\title{
Eine Klasse von 3-dimensionalen Mannigfaltigkeiten. $\mathbf{I}^{\star}$
}

\author{
Friedhelm WaLdHAUSEN (Bonn)
}

Objekt dieser Arbeit sind orientierbare kompakte 3-dimensionale Mannigfaltigkeiten, $M$, mit der Eigenschaft: Es gibt in $\stackrel{\circ}{M}$ ein System disjunkter Tori, $T$, so daß jede Komponente von $M-\stackrel{\circ}{U}(T)$ homöomorph ist zu einem Faserbündel mit $S^{1}$ als Faser $(U(T)$ bezeichnet eine reguläre Umgebung von $T$ ). Wir bezeichnen $T$ als eine Graphenstruktur für $M$, und $M$ selbst als eine Graphenmannigfaltigkeit (als Anlaß dieser Namensgebung kann man $\S 9$ betrachten).

Es ist für unsere Zwecke notwendig, eine vorgegebene Graphenstruktur zunächst in bestimmter Weise zu vereinfachen (dabei wird i.a. die Mannigfaltigkeit in eine zusammenhängende Summe zerlegt). Eine Graphenstruktur, die sich nicht weiter vereinfachen läßt, heißt reduzierte Graphenstruktur, und eine Mannigfaltigkeit mit vorgegebener reduzierter Graphenstruktur heißt reduzierte Graphenmannigfaltigkeit.

Unser Hauptergebnis ist: Ein Homöomorphismus zwischen „hinreichend komplizierten" reduzierten Graphenmannigfaltigkeiten läßt sich so deformieren, daß er die eine Graphenstruktur auf die andere abbildet; (8.1).

Spezielle Graphenmannigfaltigkeiten sind die orientierbaren unter den von SEIFERT in [12] behandelten ,gefaserten Räumen“ (Kenntnis der Seifertschen Arbeit [12] ist für das Verständnis dieser Arbeit nicht notwendig, aber nützlich); unser Ergebnis impliziert, daß jeder Homöomorphismus zwischen „hinreichend komplizierten“ orientierbaren gefaserten Räumen in einen fasertreuen Homöomorphismus deformiert werden kann; (10.1). Die von SeIFERT angegebene Klassifikation der gefaserten Räume gegenüber fasertreuen Homöomorphismen läßt sich übertragen auf die Klassifikation von reduzierten Graphenmannigfaltigkeiten gegenüber solchen Homöomorphismen, die die Graphenstruktur respektieren ( $(9)$; nach dem genannten Ergebnis ist dies „i. a.“ zugleich die topologische Klassifikation.

Die Herleitung des Hauptsatzes beansprucht den größten Teil der Arbeit; man könnte sie bezeichnen als eine Anwendung der Theorie ,inkompressibler Flächen“":

Sei $F$ ein System von (orientierbaren kompakten) Flächen in der (orientierbaren kompakten) Mannigfaltigkeit $M$ (so daß $F \cap \partial M=\partial F$ );

* Aus technischen Gründen ist die Arbeit in zwei Teile zerlegt. Der zweite Teil enthält đie Paragraphen $6-10$. 
keine Komponente von $F$ sei eine 2-Sphäre. Der Terminologie von W. HAKEN folgend nennen wir $F$ inkompressibel in $M$, wenn folgendes gilt: Ist $D$ ein 2-Element in $M$, so da $D \cap F=\partial D$, dann gibt es ein 2-Element in $F$, dessen Rand ebenfalls $\partial D$ ist.

Es gilt der (fast triviale) Satz: Sei $M$ eine irreduzible Mannigfaltigkeit. Seien $F$ und $G$ Flächensysteme in $M$ (so daß $F \cap \partial M=\partial F$ und $G \cap \partial M=$ $\partial G)$; sei $F$ isotop so deformiert worden, daß der Durchschnitt $F \cap G$ möglichst klein ist. Sei $U(G)$ eine (bezüglich $F$ kleine) reguläre Umgebung von $G$, und sei $\tilde{M}=(M-U(G))$. Dann gilt: Ist $F$ inkompressibel in $M$, dann ist $F \cap \tilde{M}$ inkompressibel in $\widetilde{M} ;(1.9 .1)$.

Wir benutzen diesen Satz, um inkompressible Flächen zu klassifizieren, insbesondere auch, um die Nicht-Existenz unerwünschter inkompressibler Flächen nachzuweisen; die Situation ist dabei folgende: $M$ ist eine Mannigfaltigkeit, die uns interessiert. In $M$ finden wir ein System von (nicht notwendig inkompressiblen) Flächen, $G$, so daß jede Komponente von $\tilde{M}=(M-U(G))$ eine Mannigfaltigkeit ist, deren inkompressible Flächen wir bereits kennen. Es genügt dann, daß wir auf der Suche nach inkompressiblen Flächen in $M$ überhaupt nur solche Flächen beachten, deren Durchschnitt mit $\tilde{M}$ ein in $\tilde{M}$ inkompressibles Flächensystem ist.

Typische Fälle sind folgende:

a) $M$ ist ein Vollring; $G$ ist eine Meridianfläche; $\tilde{M}$ ist ein 3-Element.

b) $M$ ist ein $S^{1}$-Bündel, $\partial M \neq \emptyset ; G$ ist ein System von Kreisringen; $\tilde{M}$ ist ein Vollring. ( $\$ 2$ ).

c) $M$ ist eine Graphenmannigfaltigkeit, von der wir bereits wissen, daß sie irreduzibel ist; $G$ ist die Graphenstruktur; jede Komponente von $\tilde{M}$ ist ein $S^{1}$-Bündel. ( $(7)$.

(Der Nachweis, daß eine gegebene Mannigfaltigkeit irreduzibel ist, ist per definitionem der Nachweis, daß es in dieser Mannigfaltigkeit keine inkompressible 2-Sphäre gibt; er wird mit ganz ähnlichen wie den soeben skizzierten Argumenten geführt.)

Seien nun $M$ und $N$ Graphenmannigfaltigkeiten, und sei $\varphi: N \rightarrow M$ ein Homöomorphismus. Wir suchen in $N$ eine inkompressible Fläche $F$ (die eine Komponente der Graphenstruktur ist oder auch nur in enger Beziehung zu der Graphenstruktur steht). Es ist dann $\varphi(F)$ eine inkompressible Fläche in $M$, und aus unserer Kenntnis der inkompressiblen Flächen in $M$ können wir schließen, daß $\varphi$ so deformiert werden kann, daß hinterher jedenfalls $F$ "schön" abgebildet wird. Der Beweis des Hauptsatzes besteht in einem induktiven Argument dieser Art. - Die soeben mit $F$ bezeichnete inkompressible Fläche in $N$ braucht nicht immer zu existieren; da $\beta$ sie existiert, ist i.w. unsere Bedingung, da $\beta N$, ,hinreichend kompliziert" sei. 
Da eine Graphenmannigfaltigkeit als Randflächen nur Tori haben kann, gibt es sicherlich nur ,sehr wenig“ Graphenmannigfaltigkeiten. Tatsächlich gibt es aber noch viel weniger: Sei z.B. $k$ eine Knotenlinie in der 3-Sphäre; $k$ habe keinen „Begleitknoten“ im Sinne von SCHUBERT [11]. Ist das Komplement einer offenen Umgebung von $k$ eine Graphenmannigfaltigkeit, dann ist $k$ ein Torusknoten.

Es sei noch auf eine Beziehung zur Funktionentheorie hingewiesen: Ist $X$ ein komplexer Raum der komplexen Dimension 2, und ist $x$ ein normaler singulärer Punkt in $X$, dann besitzt $x$ eine Umgebung, die homöomorph ist zum Kegel über einer 3-dimensionalen Mannigfaltigkeit. Diese 3-Mannigfaltigkeit ist eine Graphenmannigfaltigkeit, [8], [10].

Ich möchte Herrn Dr. EPSTEIN für Diskussionen danken, die für das Zustandekommen dieser Arbeit sehr nützlich waren.

\section{Inhaltsübersicht}

0 . Notationen und Verabredungen

1. Inkompressible Flächen

2. Inkompressible Flächen in $S^{1}$-Bündeln

3. Eine pathologische Darstellung des $S^{1}$-Bündels über dem Möbiusband

4. Charakterisierung der planaren unter den inkompressiblen Flächen in $S^{1}$ Bündeln

5. Homöomorphismen von $S^{1}$-Bündeln; ,natürliche Koordinatensysteme“ in den Randflächen von $S^{1}$-Bündeln

6. Definition von Graphenmannigfaltigkeiten; Reduktion von Graphenstrukturen

7. Flächen in reduzierten Graphenmannigfaltigkeiten

8. Homöomorphismen von reduzierten Graphenmannigfaltigkeiten

9. Klassifikation von ,hinreichend komplizierten" reduzierten Graphenmannigfaltigkeiten

10. Seifertsche Faserräume

\section{Notationen und Verabredungen}

Semilinearer Standpunkt. Mannigfaltigkeiten, Flächen, usw. haben eine feste semilineare Struktur. Homöomorphismen, Einbettungen, usw. sind semilineare Abbildungen. Zum Beispiel bezeichnen wir mit „einfachgeschlossene Kurve" eine semilineare Einbettung der 1-Sphäre.

Eine Mannigfaltigkeit ist eine orientierbare kompakte 3-dimensionale Mannigfaltigkeit mit oder ohne Rand. Sie ist i.a. zusammenhängend; nur werden wir manchmal ,System von Untermannigfaltigkeiten“ abkürzen durch „Mannigfaltigkeit“.

Eine Fläche ist eine zusammenhängende kompakte 2-dimensionale Mannigfaltigkeit mit oder ohne Rand. Sie ist i. a. orientierbar; die einzigen nicht-orientierbaren Flächen treten als Basisräume oder höchstens als Schnitte von Faserräumen auf. - Wird der Zusammenhang nicht behauptet, so sprechen wir von einem System von Flächen. 
Untermannigfaltigkeiten und reguläre Umgebungen sind kompakt.

Eine reguläre Umgebung ist immer ,hinreichend klein“; eine typische Konstruktion ist die folgende: Sei $X$ eine Mannigfaltigkeit oder Fläche, und $Y$ ein Unterraum von $X$. Seien $Z_{1}, \ldots, Z_{n}$ die sämtlichen (kompakten) Unterräume von $X$, die bereits in der Argumentation erwähnt wurden. Wir wählen eine (endliche) Triangulation von $X$, in der $Y$ und $Z_{1}, \ldots, Z_{n}$ Unterkomplexe sind. Der geschlossene Stern von $Y$ in der zweiten baryzentrischen Unterteilung dieser Triangulation ist dann eine reguläre Umgebung von $Y$.

Ist $X$ ein System von $n$-Mannigfaltigkeiten, dann bezeichnet $\partial X$ das System der Rand-( $n-1)$-Mannigfaltigkeiten von $X$.

Wird von einer ,Fläche $F$ in der Mannigfaltigkeit $M$ " gesprochen, und geht nicht aus dem Zusammenhang das Gegenteil hervor, so ist $F \cap \partial M=\partial F$. Sprechen wir von einer „Fläche $F$ in $M$ oder $\partial M$ ", so erlauben wir zusätzlich, daß $F$ eine Randkomponente von $M$ oder eine (zusammenhängende kompakte) 2-Untermannigfaltigkeit von $\partial M$ ist.

Ein System von Flächen in $M$ oder $\partial M$ besteht aus endlich vielen paarweise punktfremden Komponenten.

Ein Homöomorphismus $f: X \rightarrow Y$ ist surjektiv, sofern nicht ausdrücklich ein anderes Bild als $Y$ angegeben wird.

Eine isotope Deformation von $X$ ist eine niveauerhaltende Abbildung $h: X \times I \rightarrow X \times I$, so daß auf jedem Niveau $h \mid X \times t=h_{t}$ ein Homöomorphismus von $X$ auf $X$ ist, und daß $h_{0}=$ id. Wir werden ,isotope Deformation" oft abkürzen durch „Deformation".

Unterräume $Y_{1}$ und $Y_{2}$ in $X$ heißen isotop, wenn es eine isotope Deformation von $X$ gibt, $h_{t}, t \in I$, so daß $h_{1}\left(Y_{1}\right)=Y_{2}$.

Seien $F$ und $G$ Flächen in $M$ oder $\partial M$; es sei $F \cap G=\partial F=\partial G$. $F$ und $G$ heißen parallel, wenn es eine Fläche $H$ und eine Einbettung $f: H \times I \rightarrow M$ gibt, so daß $f(H \times 0)=F$ und $f(H \times 1 \cup \partial H \times I)=G$. (Sind $F$ und $G$ parallel, so gibt es auch eine Einbettung $f^{\prime}: H \times I \rightarrow M$, so daß $f^{\prime}(H \times 0$ $\cup \partial H \times I)=F$ und $f^{\prime}(H \times 1)=G$.)

Eine Fläche $F$ in $M$ heiBt rand-parallel, wenn es eine zu $F$ parallele Fläche $F^{\prime}$ in $\partial M$ gibt.

Für Kurven und Bögen in einer Fläche sind die Begriffe „parallel““ und ,rand-parallel" analog definiert.

Sei $M$ eine Mannigfaltigkeit und $F$ ein System von Flächen in $M$. Wir werden häufig die (möglicherweise nicht zusammenhängende) Mannigfaltigkeit $\tilde{M}$ zu betrachten haben, die entsteht, wenn , $M$ an $F$ aufgeschnitten" wird. Wir können uns $\tilde{M}$ vorstellen als eine Mannigfaltigkeit, die zwei Kopien von $F$ in ihrem Rand enthält, so da $B$ wir $M$ erhalten, wenn wir diese beiden Kopien identifizieren; $(F$ wird immer zweiseitig sein). Meistens ist es aber bequemer, $\tilde{M}$ als Untermannigfaltigkeit von $M$ 
anzusehen: Dazu setzen wir $\tilde{M}=(M-U(F))$; dabei ist $U(F)$ eine reguläre Umgebung von $F$ in $M$.

Sei $G$ ein weiteres System von Flächen in $M$. $G$ sei in allgemeiner Lage bezüglich $F$, (d.h. $F \cap G$ bestehe aus paarweise punktfremden einfachgeschlossenen Kurven und einfachen Bögen, deren Endpunkte in $\partial M$ liegen; jede Schnittlinie sei Durchsetzungslinie. - Wir werden später häufig allgemeine Lage annehmen, auch ohne es explizit zu sagen: Allgemeine Lage läßt sich immer herstellen mit Hilfe einer beliebig kleinen Deformation eines der beiden Systeme.) Beim Aufschneiden von $M$ an $F$ zu $\tilde{M}$ entsteht dann aus $G$ ein Flächensystem $\tilde{G}$ in $\tilde{M}$. (In der andern Formulierung: Die reguläre Umgebung $U(F)$ wird klein bezüglich $G$ gewählt; es ist dann $\widetilde{G}=G \cap \tilde{M}$.) - Im Gegensatz zu $\tilde{M}$ hängt natürlich $\tilde{G}$ sehr stark davon ab, ob und wie $F$ vor dem Aufschneiden deformiert wurde.

Eine Aussage wie ,die Kurve $k$ ist homolog zur Kurve $l$ " bezieht sich auf die von $k$ und $l$ repräsentierten Zyklen in der Homologie eines $k$ und $l$ enthaltenden Raumes (der angegeben wird, wenn ein Zweifel möglich ist); Koeffizienten sind ausnahmslos die ganzen Zahlen. Werden $k$ und $l$ nicht als orientierte Kurven angegeben und ist es nicht aus irgendeinem Grunde selbstverständlich, daß von orientierten Kurven die Rede ist, dann bedeutet „homolog": homolog bis evtl. aufs Vorzeichen.

Es wird oft von ,dem" $S^{1}$-Bündel über einer Fläche $F, \partial F \neq \emptyset$, z. B. dem Möbiusband, die Rede sein: Wir betrachten nur solche $S^{1}$-Bündel, die orientierbaren Totalraum haben, und von diesen gibt es über einer berandeten Fläche bis auf Äquivalenz nur eines.

Die folgenden Symbole werden im angegebenen (und nur in diesem) Sinn benutzt:

$$
\begin{array}{ll}
I=[0,1] & \text { Einheitsintervall } \\
D & \text { 2-Element } \\
E & \text { 3-Element } \\
U(\ldots) & \text { reguläre Umgebung von ... }
\end{array}
$$

„Abgeschlossene Hülle“ und „Inneres" bezeichnen wir in der üblichen Weise; bei längeren Ausdrücken schreiben wir (...) und (...), bzw. $\stackrel{\circ}{U}(.$.$) .$

Eckige Klammern bezeichnen abgeschlossene Intervalle, Literaturhinweise und Homologieklassen.

Die folgenden Begriffe sind an folgenden Stellen definiert: ,vertikal“, „,horizontal"“ in (2.5); ,,irreduzibel" in (1.7); ,,reduziert" in (6.2).

\section{Inkompressible Flächen}

(1.1) Sei $M$ eine Mannigfaltigkeit, und $F$ ein System von Flächen in $M$ oder $\partial M$. $F$ heißt ,kompressibel in $M$ ", wenn einer der beiden folgenden Fälle vorliegt: 
(1.1.1) Es gibt eine einfach-geschlossene Kurve $k$ in $\stackrel{\circ}{F}$, die nicht ein 2-Element in $F$ berandet, und eine Einbettung des 2-Elements $D$ in $M$, so $\mathrm{da} \beta \stackrel{\circ}{D} \subset \stackrel{\circ}{M}$ und $D \cap F=k$.

(1.1.2) Es gibt eine Einbettung des 3-Elements $E$ in $M$, so daß $E \cap F$ $=\partial E$.

(1.2) Sei $M$ eine Mannigfaltigkeit, $G$ ein System von Flächen in $\partial M$, und $F$ ein System von Flächen in $M . F$ heißt ,rand-kompressibel in $M$ bezüglich $G^{\star 6}$, wenn einer der beiden folgenden Fälle vorliegt:

(1.2.1) Es gibt einen Bogen $l$ in $F$, der nicht zu einem Bogen in $\partial F$ parallel ist, und eine Einbettung des 2-Elements $D$ in $M$, so daß

und $D \cap F=l$.

$$
D \cap(F \cup G)=\partial D \text {, }
$$

(1.2.2) Es gibt eine Randkurve von $F$, die ein 2-Element in $G$ berandet.

Ist $F$ nicht kompressibel in $M$, so nennen wir $F$, inkompressibel in $M$ ". Besteht kein Zweifel über die Mannigfaltigkeit, so werden wir statt „inkompressibel in $M^{\text {" }}$ auch ,inkompressibel“ sagen.

Ist $F$ nicht rand-kompressibel in $M$ bezüglich $G$, so nennen wir $F$

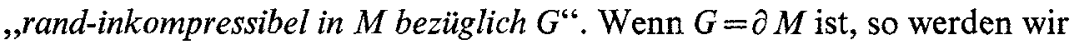
dies abkürzen durch ,rand-inkompressibel in $M$ “. Besteht außerdem kein Zweifel über die Mannigfaltigkeit, so werden wir kurz „randinkompressibel" sagen.

(1.3) Lemma. Sei $F$ ein inkompressibles System von Flächen in $M$ oder $\partial M$. Jede Randkurve von $F$, die ein 2-Element in $\partial M$ berandet, berandet ein 2-Element in $F$.

Beweis. Seien $D_{1}, D_{2}, \ldots, D_{n}$ die sämtlichen Komponenten von $F$, die 2-Elemente sind. Auf dem Rande einer regulären Umgebung

$$
U\left(\partial M \cup D_{1} \cup \cdots \cup D_{n}\right)
$$

sei $G$ die Teilfläche $G=(\stackrel{\circ}{M} \cap \partial U)$. Angenommen, es gäbe Randkurven von $F$, die in $\partial M$, aber nicht in $F$ ein 2-Element beranden: Sei $k$ eine innerste von diesen, und sei $F^{\prime}$ die $k$ enthaltende Komponente von $F$. Es folgt, daß $F$ kompressibel ist, da die nächst $k$ liegende Schnittkurve aus $F^{\prime} \cap G$ auf $G$ ein 2-Element berandet, das $F$ sonst nicht trifft.

(1.4) Lemma. Sei F ein System von Flächen in dem 3-Element E. Jede Komponente von $F$, die ein 2-Element ist, ist parallel zu zwei 2-Elementen in $\partial E$, und ist insbesondere rand-kompressibel. Gibt es eine Komponente von $F$, die nicht ein 2-Element ist, dann ist $F$ kompressibel.

Beweis. $z u$ 1) Es folgt aus dem Schoenflies-Satz, [1], daß $E$ von einem 2-Element in zwei 3-Elemente zerlegt wird. Die Behauptung folgt nun daraus, daß zwei 2-Elemente $D$ und $D^{\prime}$ in $\partial E$, mit $D \cap D^{\prime}=\partial D=\partial D^{\prime}$, parallel sind. 
$z u$ 2) Es genügt, den Beweis für den Fall zu führen, daß keine Komponente von $F$ ein 2-Element ist. - Ist jede Komponente von $F$ eine 2-Sphäre, so folgt aus dem Schoenflies-Satz, daß es eine innerste gibt, auf die (1.1.2) zutrifft.

Sei also $F^{\prime}$ eine Komponente, die keine 2-Sphäre (und kein 2-Element) ist. Ist $\partial F^{\prime} \neq \emptyset$, so ist $F$ nach (1.3) kompressibel. Ist $\partial F^{\prime}=\emptyset$, so ist nach dem verallgemeinerten Satz von Alexander, [4], $F^{\prime}$ kompressibel: Es gibt also ein 2-Element $D$ in $E$, mit $D \cap F^{\prime}=\partial D$, so da $\partial D$ nicht ein 2-Element auf $F^{\prime}$ berandet. Nach einer kleinen Deformation von $D$, falls notwendig, besteht $D \cap F$ aus paarweise disjunkten einfach-geschlossenen Kurven. Gibt es 2-Elemente in $F$, deren Rand eine solche Schnittkurve ist, so sei $D^{\prime}$ ein innerstes von diesen: Wir ersetzen durch $D^{\prime}$ das von $\partial D^{\prime}$ auf $D$ berandete 2-Element und heben $D^{\prime}$ von $F$ ab; die Anzahl der Schnittkurven $D \cap F$ wird dabei kleiner. - Ist keine der Schnittkurven Rand eines 2-Elements auf $F$, dann zeigt irgendeine auf $D$ innerste, da $B$ (1.1.1) zutrifft.

(1.5) Lemma. Sei $M$ eine Mannigfaltigkeit, und sei F ein System von Flächen in $M$ oder $\partial M$. F ist genau dann inkompressibel, wenn jede Komponente von $F$ inkompressibel ist.

Beweis. Eine Richtung ist trivial; wir beweisen die andere, indem wir die beiden Fälle (1.1.1) und (1.1.2) nachprüfen: Sei $F^{\prime}$ eine Komponente von $F ; F^{\prime}$ sei kompressibel. $Z u$ zeigen ist, daß $F$ kompressibel ist.

$z u$ (1.1.1): Der Beweis ist identisch mit einem Argument aus dem Beweis von (1.4).

$z u$ (1.1.2): Ist $F^{\prime}=\partial E$, und $\stackrel{\circ}{E} \cap F \neq \emptyset$, so folgt die Behauptung aus (1.4).

Eine ähnliche Situation wie in dem folgenden Lemma werden wir noch oft antreffen: In der Mannigfaltigkeit $M$ sind $F$ und $G$ zwei Systeme von Flächen, die einander in allgemeiner Lage schneiden. $U(F)$ ist eine reguläre Umgebung von $F$, die klein ist bezüglich $G . \tilde{M}$ ist die Untermannigfaltigkeit $(M-U(F))$, und in $\tilde{M}$ ist $\tilde{G}$ das Flächensystem $\tilde{M} \cap G$. (Um umständliche Formulierungen zu vermeiden, lassen wir hier zu, daß $\tilde{M}$ unzusammenhängend ist.)

(1.6) Lemma. In der Mannigfaltigkeit M sei F ein System von Flächen und $S$ eine inkompressible 2-Sphäre. $S$ sei so gewählt, daß die Anzahl der Schnittkurven $S \cap F$ möglichst klein ist. Dann ist $\tilde{S}=S \cap \tilde{M}$ inkompressibel in $\tilde{M}=(M-U(F))$.

Beweis. Die Behauptung sei falsch: Dann gibt es ein 2-Element $D$ in $\tilde{M}$, so daß $D \cap \tilde{S}=\partial D$ kein 2-Element auf $\tilde{S}$ berandet. Auf $S$ berandet $\partial D$ zwei 2-Elemente, $D_{1}$ und $D_{2} . D \cup D_{j}, j=1,2$, ist eine 2-Sphäre, die kleineren Durchschnitt mit $F$ hat, und daher nach Voraussetzung über 
$S$ ein 3-Element $E_{j}$ berandet. Es ist $\partial E_{1} \cap \partial E_{2}=D$, und entweder $\check{E}_{1} \cap \mathscr{E}_{2}=\emptyset$, oder, etwa, $E_{1} \subset E_{2}$. Es folgt, daß $S$ entweder das 3-Element $E_{1} \cup E_{2}$ oder das 3-Element $\left(E_{2}-E_{1}\right)$ berandet.

(1.7) Definition. Die Mannigfaltigkeit $M$ heißt ,irreduzibel“, wenn es in $M$ keine inkompressiblen 2-Sphären gibt.

(1.8) Satz. In der Mannigfaltigkeit $M$ sei $F$ ein System inkompressibler Flächen. $\tilde{M}=(M-U(F))$ ist genau dann irreduzibel, wenn $M$ irreduzibel ist.

Beweis. 1) $\tilde{M}$ sei nicht irreduzibel. Dann gibt es eine 2-Sphäre im Innern von $\tilde{M}$, die in $\tilde{M}$ kein 3-Element berandet. Angenommen, sie berandete in $M$ das 3-Element $E$ : Das hieße aber, daß im Innern von $E$ eine geschlossene Komponente von $F$ läge, und nach (1.4) wäre $F$ nicht inkompressibel. 2) $M$ sei nicht irreduzibel. Es sei $S$ eine inkompressible 2-Sphäre in $M$, deren Durchschnitt mit $F$ aus möglichst wenig disjunkten einfach-geschlossenen Kurven besteht. Wir haben zu zeigen, daß $S \cap F=\emptyset$; angenommen, es sei $S \cap F \neq \emptyset$. Unter den Schnittkurven gibt es solche, die auf $S$ innerste Schnittkurven sind. Da $F$ inkompressibel ist, gibt es also Schnittkurven, die auf $F$ ein 2-Element beranden. Sei $D$ ein 2-Element in $F$, das von einer Schnittkurve berandet wird, und in dessem Innern keine Schnittkurve liegt: Die Existenz von $D$ führt zu einem ähnlichen Widerspruch wie in (1.6).

(1.9) Satz. Die Mannigfaltigkeit $M$ sei irreduzibel. $F$ und $G$ seien zwei Systeme von Flächen in $M$. $G$ sei inkompressibel in $M$, und $G$ sei so defor-

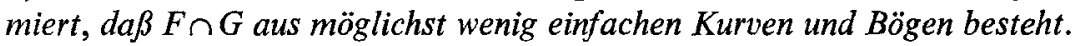

Es sei $\tilde{M}=(M-U(F))$, und $\tilde{G}=G \cap \tilde{M}$, Dann gilt:

(1.9.1) $\tilde{G}$ ist inkompressibel in $\tilde{M}$.

(1.9.2) Keine berandete Komponente von $\tilde{G}$ ist parallel zu einer Teilfläche von $(\partial \tilde{M}-\partial M)$.

(1.9.3) Es ist möglich, daß $\tilde{G}$ rand-kompressibel bezüglich $(\partial \tilde{M}-\partial M)$ ist: Aber wenn das so ist, dann liegen die beiden Endpunkte eines definierenden Bogens in derselben Randkurve von $\tilde{G}$.

Beweis. $z u$ 1) Angenommen, die Behauptung sei falsch. Dann gibt es im Innern von $\tilde{M}$ ein 2-Element $D$, so daß $D \cap \tilde{G}=\partial D$ kein 2-Element in $\tilde{G}$ berandet. $D a G$ inkompressibel in $M$ ist, berandet $\partial D$ ein 2-Element $D^{\prime}$ in $G$. Die 2-Sphäre $D \cup D^{\prime}$ ist, da $M$ irreduzibel ist, Rand eines 3-Elements $E$ in $\stackrel{\circ}{M}$. Also können wir $G$ so deformieren, daß alle auf $D^{\prime}$ liegenden Schnitte mit $F$ verschwinden und daß neue nicht hinzukommen, im Widerspruch zur Voraussetzung.

$z u$ 2) $F$ ist zweiseitig in $M$; das Weitere ist offensichtlich.

$z u$ 3) rand-kompressibel nach (1.2.2) führt auf den vorigen Fall, da $F$ inkompressibel und $M$ irreduzibel ist. - Also nehmen wir an, es gibt 
ein 2-Element $D$ in $\tilde{M}$, so da $D \cap(\tilde{G} \cup \partial \tilde{M})=\partial D$ und daß $D \cap \tilde{G}=l$ ein Bogen ist, dessen Endpunkte in verschiedenen Randkurven von $\widetilde{G}$ liegen. Es gibt dann eine Deformation von $G$ in der Nähe von $D$, deren Effekt ist, daß $l$ bei festgehaltenen Endpunkten über $D$ nach $D \cap \partial \widetilde{M}$, und noch ein bißchen weiter wandert; dabei wird die Anzahl der Schnittkurven $G \cap \partial U(F)$ verringert. Da nach Voraussetzung $F$ und $G$ einander in allgemeiner Lage schneiden, und da $U(F)$ klein ist bezüglich $G$, folgt, daß es eine Deformation von $G$ gibt, so daß die Anzahl der Schnittkurven $G \cap F$ verringert wird, im Widerspruch zur Voraussetzung.

(1.10) Lemma. Sei $M$ eine irreduzible Mannigfaltigkeit. Jede Randfläche von $M$ sei ein Torus. In $M$ sei $G$ eine inkompressible Fläche; $G$ sei nicht ein 2-Element; $G$ sei rand-kompressibel. Dann ist $G$ ein rand-paralleler Kreisring.

Beweis. Da $G$ inkompressibel ist und nicht ein 2-Element, ist keine der Randkurven von $G$ auf $\partial M$ zusammenziehbar. Sei also $D$ ein 2-Element, so daß $D \cap(G \cup \partial M)=\partial D$ und da $D \cap G=l$ ein nicht rand-paralleler Bogen in $G$ ist.

1. Fall. Beide Endpunkte von l liegen in der Randkurve $k$ von $G$. Seien $k_{1}$ und $k_{2}$ Kurven in $G$, so daß es eine Umgebung von $k \cup l$ in $G$ gibt, deren Rand $k \cup k_{1} \cup k_{2}$ ist, und seien $k_{1}^{\prime}$ und $k_{2}^{\prime}$ Kurven in $\partial M$, die analog durch $k$ und $D \cap \partial M$ definiert sind. $k, k_{1}^{\prime}, k_{2}^{\prime}$ sind gegenseitig punktfremde Kurven auf einem Torus, die zusammen beranden: Es folgt, da $B$ mindestens eine von ihnen, etwa $k_{1}^{\prime}$, ein 2-Element $D_{1}^{\prime}$ auf $\partial M$ berandet; es ist $k \cap D_{1}^{\prime}=\emptyset$, da $k$ kein 2-Element berandet. - Indem wir, grob gesprochen, $D_{1}^{\prime}$ mit $D$ zusammensetzen, finden wir ein 2-Element $D_{1}$ im Innern von $M$, so daß $D_{1} \cap G=k_{1}$. Da $G$ inkompressibel ist, berandet $k_{1}$ ein 2-Element auf $G$, und es folgt, daß im Gegensatz zu unserer Annahme $l$ rand-parallel ist.

2. Fall. Die Endpunkte von l liegen in den Randkurven $k$ und $k^{\prime} . k$ und $k^{\prime}$ sind disjunkt und nicht zusammenziehbar, sie beranden daher zwei Kreisringe auf $\partial M$; sei $G^{\prime}$ derjenige, der $D \cap \partial M$ enthält. Da keine Randkurve von $G$ ein 2 -Element auf $\partial M$ berandet, ist $G \cap G^{\prime}=k \cup k^{\prime}$. Sei $U\left(G^{\prime} \cup D\right)$ eine reguläre Umgebung von $G^{\prime} \cup D$ in $M$. Der Durchschnitt $G^{\circ} \cap \partial U\left(G^{\prime} \cup D\right)$ besteht aus genau einer Kurve. Sie berandet ein 2-Element in $\partial U$ und daher auch in $G$. Die Eulersche Charakteristik von $G \cap U\left(G^{\prime} \cup D\right)$ ist -1 , also hat $G$ die Charakteristik 0 . Da G orientierbar ist, ist $G$ folglich ein Kreisring. Sei nun $U(D)$ eine reguläre Umgebung von $D$ in $M$. Wir betrachten die 2-Sphäre, die zusammengesetzt ist aus dem Kreisring $\left(\left(G \cup G^{\prime}\right)-U(D)\right)$ und den von seinen Randkurven auf $\partial U(D)$ berandeten 2-Elementen. $\mathrm{Da} M$ irreduzibel ist, berandet diese 2-Sphäre ein 3-Element in $M$ : Es folgt, daß $G$ und $G^{\prime}$ parallel sind. 


\section{Inkompressible Flächen in $\boldsymbol{S}^{\mathbf{1}}$-Bündeln}

(2.1) Definition. Ein Vollring ist homöomorph zu $D \times S^{1}$. - Ein rand-inkompressibles 2-Element in einem Vollring heißt, ,Meridianfläche“.

Wird $D \times S^{1}$ aufgeschnitten an $D \times y, y \in S^{1}$, so entsteht ein 3-Element. Ein 2-Element ist inkompressibel: also folgt aus (1.8), daß ein Vollring irreduzibel ist.

(2.2) Lemma. In dem Vollring $M$ seien $F$ und $G$ zwei Systeme von Meridianflächen, die gleich viele Komponenten haben. Dann gibt es eine Deformation von $M$, die $F$ in $G$ überführt. Ist $\partial F \subset F \cap G$, dann kann die Deformation so gewählt werden, daß sie auf $\partial M$ konstant ist.

Beweis. 1) Seien $F_{1}, \ldots, F_{n}$ und $G_{1}, \ldots, G_{n}$ die Komponenten von $F$ und $G$. Es ist $\operatorname{ker}\left(H_{1}(\partial M) \rightarrow H_{1}(M)\right)$ frei zyklisch. Also folgt, daß die $\partial F_{j}$ in $\partial M$ paarweise parallel sind, ebenso die $\partial G_{j}$; und die $\partial F_{j}$ sind isotop in $\partial M$ zu den $\partial G_{j}$. Daher gibt es eine Deformation von $F$, so daB hinterher $\partial F \cap \partial G=\partial F=\partial G$, daß ferner $\stackrel{\varrho}{U}(\partial M) \cap F \cap G=\emptyset$ für eine Umgebung von $\partial M$, und da $F \cap G$ aus einfach-geschlossenen Kurven besteht. - War bereits zu Anfang $\partial F \subset F \cap G$, dann kann die Deformation so gewählt werden, daß sie auf $\partial M$ konstant ist.

2) Sei $k$ eine Schnittkurve aus $F \cap G$. Sei $D$ das von $k$ in $G$ berandete 2-Element; sei $k$ so gewählt, daß $D_{D} \cap F=\emptyset$. $k$ berandet ein 2-Element $D^{\prime}$ in $F . D \cup D^{\prime}$ ist eine 2-Sphäre und Rand eines 3-Elements $E$ in $M$.

a) $k \subset \stackrel{G}{G}$ : Wir deformieren $F$ so, daß $D^{\prime}=D$ wird, und heben dann ab; das geht mit einer Deformation in einer Umgebung von $E$. Dabei verschwinden die Schnitte von $D^{\prime}$ mit $G$, insbesondere $k$.

b) $k \subset \partial G$ : Wir deformieren $F$ so, daß $D^{\prime}=D$ wird. Das geht mit einer Deformation, die konstant ist auf $\partial M$, und die frühere Normierungen des Typs (b) nicht stört.

(2.3) Lemma. Sei $M$ ein Vollring. G sei eine inkompressible Fläche in $M ; G$ sei nicht ein 2-Element. Dann ist $G$ ein rand-paralleler Kreisring.

Beweis. Sei $F$ eine Meridianfläche in $M$. Da $\partial M$ ein Torus ist, können wir $G$ so deformieren, daß seine Randkurven $\partial F$ „,monoton“ schneiden, d.h. daß für eine Randkurve $k$ von $G$ die Anzahl der Schnittpunkte $k \cap \partial F$ gleich ist dem Betrag der Schnittzahl von $k$ und $\partial F$ in $\partial M$. Anschließend deformieren wir $G$ so, wie im Beweis von (1.9) beschrieben, daß $G \cap F$ aus möglichst wenig einfachen Kurven und Bögen besteht. Das kann die Situation in $\partial M$ nicht wesentlich ändern, da der Effekt wäre, die Anzahl der Schnittpunkte $\partial G \cap \partial F$ zu verringern, was nicht geht. - Es ist nicht möglich, daß $G \cap F=\emptyset$, da dann $G$ ein 2-Element sein müßte. Keiner der Schnitte $G \cap F$ ist eine geschlossene Kurve, da eine auf $F$ innerste nach (1.9.1) auf $G$ ein von weiteren Schnitten freies 2-Element beranden müßte, 
und da dies wiederum parallel wäre zu einem Teil von $F$, im Widerspruch zu (1.9.2). - Für eine Kurve in $\partial M$ ist die Schnittzahl in $M$ mit dem relativen 2-Zykel $F$ bis evtl. aufs Vorzeichen gleich der Schnittzahl in $\partial M$ mit $\partial F$ : Also ist keiner der Bögen $G \cap F$ rand-parallel in $G$. Ein auf dem 2-Element $F$ äußerster dieser Bögen zeigt nun, daß $G$ rand-kompressibel ist. Die Behauptung folgt aus (1.10).

(2.4) Lemma. In dem Vollring $M$ seien $G_{1}, \ldots, G_{n}$ und $G_{1}^{\prime}, \ldots, G_{n}^{\prime} z w e i$ Systeme von Kreisringen. Es sei $\partial G_{j} \subset G_{j} \cap G_{j}^{\prime}, j=1, \ldots, n$. Eine Randkurve dieser Kreisringe habe mit der Randkurve einer Meridianfläche die Schnittzahl eins. Dann gibt es eine auf $\partial M$ konstante Deformation, die die $G_{j}$ in die $G_{j}^{\prime}$ überführt.

Beweis. Sei $F$ eine Meridianfläche. Ähnlich wie im vorangegangenen Beweis erreichen wir, daB die Durchschnitte $F \cap G_{j}$ und $F \cap G_{j}^{\prime}$ je aus genau einem Bogen bestehen. Das kann so gemacht werden, daß in der Nähe von $\partial M$ nur $F$ bewegt wird und nicht die $G_{j}$ und $G_{j}^{\prime}$. Da es für jedes $j$ nur je einen Bogen $F \cap G_{j}$ und $F \cap G_{j}^{\prime}$ gibt, haben die Bögen $F \cap G_{j}$ und $F \cap G_{j}^{\prime}$ dieselben Endpunkte (an dieser Stelle wird die Bedingung über die Schnittzahl wirklich ausgenutzt). Also können wir die $G_{j}$ so deformieren, daß $G_{j} \cap U(F)=G_{j}^{\prime} \cap U(F)$ in einer Umgebung $U(F)$ von $F$ in $M$. Betrachten wir nun $\tilde{M}=(M-U(F))$ und die $\tilde{G}_{j}=G_{j} \cap \tilde{M}$ und $\tilde{G}_{j}^{\prime}=G_{j}^{\prime} \cap \tilde{M}$, so sehen wir, daß unsere Frage zurückgeführt ist auf die Frage, ob in einem 3-Element zwei Systeme von 2-Elementen mit gemeinsamem Rand ineinander übergeführt werden können mit Hilfe einer Deformation, die auf dem Rand konstant ist.

(2.5) Definition. Mit , $p: M \rightarrow B$ “ bezeichnen wir eine Mannigfaltigkeit, die als $S^{1}$-Bündel mit $B$ als Basisraum und $p$ als Bündelprojektion gefasert ist. $B$ ist eine kompakte, nicht notwendig orientierbare 2-Mannigfaltigkeit mit oder ohne Rand.

Ein Unterraum $X$ in $M$ heißt ,vertikal ", wenn $X=p^{-1}(p(X))$.

Ein Unterraum $X$ in $M$ hei $\beta$ t, ,horizontal ", wenn $p \mid X$ eine Einbettung ist.

(2.6) Lemma. $p: M \rightarrow B$ sei ein $S^{1}$-Bündel. B sei nicht das 2-Element. Dann ist $\partial M$ inkompressibel.

Beweis. Sei zunächst $B$ orientierbar. Da $\partial B \neq \emptyset$, ist $M$ ein Produktbündel, $M=B \times S^{\mathbf{1}}$. Da $B$ nicht das 2-Element ist, ist für eine Randkurve $k$ von $B, \operatorname{ker}\left(H_{1}(k) \rightarrow H_{1}(B)\right)=0$, also ist für eine Randfläche $K$ von $M$ ebenfalls $\operatorname{ker}\left(H_{1}(K) \rightarrow H_{1}(M)\right)=0 . K$ ist ein Torus, daher $H_{1}(K) \approx$ $\pi_{1}(K)$; also ist $\operatorname{ker}\left(\pi_{1}(K) \rightarrow \pi_{1}(M)\right)=0$.

Ist $B$ nicht-orientierbar, dann sei $B^{\prime}$ die zweiblättrige orientierbare Uberlagerungsfläche; $B^{\prime}$ ist kein 2-Element. Es gibt ein Bündel $p^{\prime}: M^{\prime} \rightarrow B^{\prime}$, so daß $M^{\prime}$ eine zweiblättrige Überlagerung von $M$ ist und daß das offen- 
sichtliche Diagramm kommutativ ist. Da ein rand-inkompressibles 2Element in $M$ geliftet würde $z \mathfrak{u}$ einem rand-inkompressiblen 2-Element in $M^{\prime}$, ist damit dieser Fall auf den vorigen zurückgeführt.

(2.7) Lemma. $p: M \rightarrow B$ sei ein $S^{1}$-Bündel. B sei nicht die 2-Sphäre oder projektive Ebene. Dann ist $M$ irreduzibel.

Beweis. Sei zunächst $M$ geschlossen, dann gibt es nach unserer Voraussetzung über $B$ in $B$ eine einfach-geschlossene zweiseitige Kurve $k$, die nicht ein 2-Element in $B$ berandet. Es folgt aus (2.6), daß der Torus $p^{-1}(k)$ inkompressibel in $M$ ist. Mit (1.8) ist daher die Behauptung auf den Fall $\partial M \neq \emptyset$ zurückgeführt.

Sei also $\partial M \neq \emptyset$. Seien $c_{1}, \ldots, c_{n}$ disjunkte Bögen in $B$, die $B$ in ein (und nur ein) 2-Element aufschneiden, $\left(c_{j} \cap \partial B=\partial c_{j}\right)$. Sei $C_{j}=p^{-1}\left(c_{j}\right)$. Das System von vertikalen Kreisringen $C=C_{1} \cup \cdots \cup C_{n}$ ist inkompressibel in $M$. Denn sei etwa $D$ ein 2-Element in $\stackrel{\circ}{\text { mit }} D \cap C=\partial D$ und, etwa, $\partial D$ in $C_{n}$. Wäre $\partial D$ nicht Rand eines 2-Elements auf $C_{n}$, dann wäre es parallel zu den Randkurven von $C_{n}$, also könnten wir $D$ mit einem der Teile von $C_{n}-\partial D$ zusammensetzen zu einem rand-inkompressiblen 2-Element in $M$, im Widerspruch zu (2.6). - Sei $U(C)$ eine reguläre Umgebung von $C$ in $M ; U(C)$ sei vertikal. Dann ist $\tilde{M}=(M-U(C))$ ein $S^{1}$-Bündel über dem 2-Element, also ein Vollring. $\tilde{M}$ ist irreduzibel nach der Bemerkung (2.1), daher $M$ nach (1.8).

(2.8) Satz. $p: M \rightarrow B$ sei ein $S^{1}$-Bündel. B sei nicht die 2-Sphäre oder projektive Ebene.

$G$ sei ein System von inkompressiblen (orientierbaren) Flächen in $M$. Keine berandete Komponente von $G$ sei rand-parallel.

Dann gibt es eine isotope Deformation, nach deren Ausführung entweder (2.8.1) oder (2.8.2) gilt:

(2.8.1) $G$ ist vertikal. Insbesondere ist jede Komponente von $G$ ein Kreisring oder Torus.

(2.8.2) $p \mid G$ ist eine Überlagerungsabbildung.

Beweis. Wir behandeln zunächst den Fall $\partial M \neq \emptyset$. - Die Normierung von $G$ geschieht in mehreren Schritten.

1. Schritt. In der Nähe von $\partial M$ wird $G$ so deformiert, daß $\partial G$ straffgezogen wird; das Resultat können wir folgendermaßen beschreiben: Die Randfläche $K$ von $M$ sei dargestellt als Quotientenraum der Euklidischen $x, y$-Ebene nach dem Gitter mit ganzzahligen Koordinaten, und zwar so, daß die Projektion $p \mid K$ überlagert wird von der Projektion auf die $x$-Achse. Dann wird jede Kurve $G \cap K$ von einer Geraden überlagert.

2. Schritt. Sei $C$ ein inkompressibles System von vertikalen Kreisringen wie im Beweis von (2.7); (wir nehmen im folgenden an, daß $C \neq \emptyset$, 
da $\beta$ also $M$ kein Volling ist). Wir reduzieren den Durchschnitt $G \cap C$ mit Hilfe der in (1.9) beschriebenen Deformationen. Da $\partial G \cap \partial C$ sicher nicht mehr verkleinert werden kann, können diese Deformationen so gewählt werden, daß sie auf $\partial M$ nichts ändern. Es folgt aus (1.9), daß es dann in $G \cap C$ keine Schnittkurve gibt, die auf $C$ oder $G$ ein 2-Element berandet. Wir behaupten weiter:

Ist l ein Bogen aus $G \cap C$, so liegen die Endpunkte von l in verschiedenen Randkurven von $C$. - Denn sei die Behauptung falsch. Dann sei $D$ das 2-Element in $C$ mit $\partial D \subset l \cup \partial C$. Wenn nötig, gehen wir zu einem andern $l$ und andern $D$ über, es gilt dann $D \cap G=l$. Nach (1.10) und einer unserer Voraussetzungen ist $G$ rand-inkompressibel in $M$. Daher gibt es ein 2-Element $D^{\prime}$ in $G$ mit $\partial D^{\prime} \subset l \cup \partial G$. Da $D^{\prime} \cap C$ keine geschlossenen Kurven enthält, gibt es ein 2-Element $D^{\prime \prime}$ in $D^{\prime}$, so daß $D^{\prime \prime} \cap(C \cup \partial M)$ $=\partial D^{\prime \prime}$ und da $D^{\prime \prime} \cap C$ ein einziger Bogen ist. $D^{\prime \prime}$ kann nicht Meridianfläche sein in dem aus $M$ durch Aufschneiden an $C$ entstehenden Vollring, da es $C$ nur in einem Bogen trifft: Das zeigt aber, daß die Normierung aus dem ersten Schritt nicht vollständig ist.

3. Schritt. Nach dem vorigen Schritt besteht $G \cap C$ nur aus geschlossenen Kurven (im Innern von $C$ ), die zu Randkurven von $C$ parallel sind, und aus Bögen, die nicht rand-parallel sind. Also können wir $G$ so deformieren, daß $G \cap C$ bei festgehaltenem $G \cap \partial C$ straffgezogen wird. Hinterher besteht $G \cap C$ aus vertikalen geschlossenen Kurven und aus horizontalen Bögen.

4. Schritt. Sei $U(C)$ eine (bezüglich $G$ kleine) reguläre Umgebung von $C$ in $M . U(C)$ sei vertikal. Sei $\tilde{M}=\overline{(} M-U(C))$, und sei $\tilde{G}=G \cap \tilde{M}$.

Die Normierung aus dem dritten Schritt sei auf $U(C)$ ausgedehnt: $G \cap U(C)$ besteht dann aus vertikalen Kreisringen und aus horizontalen 2-Elementen.

Nach (1.9) und (2.3) ist jede Komponente von $\tilde{G}$ entweder ein 2-Element oder ein Kreisring. War jede Randkurve von $G$ vertikal oder war $G$ geschlossen, so ist jetzt jede Randkurve von $\tilde{G}$ vertikal, und $\tilde{G}$ besteht daher nur aus Kreisringen; da es in $\tilde{M}$ sicher ein System vertikaler Kreisringe gibt zu vorgegebenem vertikalen Randsystem, ist in diesem Falle mit (2.4) die Behauptung bewiesen.

Wir nehmen also im folgenden an: es gibt eine Randkurve von $G$, die in $\partial M$ nicht homolog ist zu einer vertikalen Kurve. Es gibt also eine Randkurve $k$ von $\tilde{G}$, die nicht vertikal ist. Nach den Normierungen im ersten und dritten Schritt trifft $k$ die Kreisringe $\tilde{M} \cap U(C)$ und $\partial \tilde{M} \cap \partial M$ nur in horizontalen Bögen. Daraus folgt, da $B$ nicht homolog null ist in $\partial \tilde{M}$. Dasselbe gilt für alle andern Randkurven von $\tilde{G}$, die nicht vertikal sind; da $\partial \tilde{M}$ ein Torus ist und da die Randkurven einander nicht schnei- 
den, gilt es für alle Randkurven von $\tilde{G}$. Folglich sind die Randkurven von $\tilde{G}$ in $\partial \tilde{M}$ sämtlich untereinander parallel. - Wir behaupten weiter:

Jede Komponente von $\tilde{G}$ ist ein 2-Element, also eine Meridianfläche in $\tilde{M}$. - Denn sei etwa $L$ ein Kreisring. Nach (2.3) ist $L$ parallel zu einem Kreisring $L^{\prime}$ in $\partial \tilde{M}$. Sei $\tilde{l}$ eine vertikale Kurve im Innern eines der Kreisringe $\tilde{M} \cap U(C)$, und sei $l$ eine Komponente von $\tilde{l} \cap L^{\prime} ; l$ ist ein nicht randparalleler Bogen in $L^{\prime}$. Da $L$ und $L^{\prime}$ parallel sind, gibt es ein 2-Element $D$ in $\tilde{M}$ mit $D \cap\left(L \cup L^{\prime}\right)=\partial D$ und $D \cap L^{\prime}=l$. Gibt es unter den Schnitten $D \cap \tilde{G}$ geschlossene Kurven, so sind sie zusammenziehbar auf $\tilde{G}$, also können wir eine auf $\tilde{G}$ innerste aufsuchen und das von ihr auf $D$ berandete 2-Element ersetzen durch das von ihr auf $\widetilde{G}$ berandete 2-Element; also dürfen wir annehmen, es gibt keine. Wegen der Wahl von $l$ und der Normierung von $\partial \widetilde{G}$ kann kein Bogen $D \cap \tilde{G}$ rand-parallel auf $\tilde{G}$ sein: Wir suchen einen $l$ zunächst liegenden dieser Bögen auf und finden, daß $\widetilde{G}$ rand-kompressibel bezüglich $\tilde{M} \cap U(C)$ ist, im Widerspruch zu (1.9.3).

$p$ ist bereits lokal homöomorph auf $G \cap(U(C) \cup \partial M)$, insbesondere auf $\partial \widetilde{G}$. Da eine vertikale Kurve auf $\partial \tilde{M}$ mit der Randkurve einer Meridianfläche die Schnittzahl eins hat, müssen die Randkurven von $\tilde{G}$ sogar horizontal sein. Also können wir ein System von horizontalen Meridianflächen finden, dessen Rand $\partial \widetilde{G}$ ist. Und nach (2.2) ist $\widetilde{G}$ isotop zu diesem System unter einer Deformation, die auf $\partial \tilde{M}$ konstant ist.

Sei nun $M$ geschlossen. Sei $K$ ein inkompressibler vertikaler Torus in $M$ wie in (2.7). Sei $G$ so deformiert, da $G \cap K$ aus möglichst wenig einfachgeschlossenen Kurven besteht. Anschließend werde $G$ so deformiert, daß dabei $G \cap K$ in $K$ straffgezogen wird, analog dem ersten Schritt oben.

Sei $U(K)$ eine vertikale reguläre Umgebung von $K$ in $M$ (klein bezüglich $G$ ). Sei $\tilde{M}=(M-U(K))$, und $\widetilde{G}=G \cap \tilde{M}$.

Nach (1.9) sind wir mit $\tilde{M}$ und $\tilde{G}$ genau in den Voraussetzungen für den berandeten Fall. Da bei der Normierung von $\tilde{G}$ in $\tilde{M}$ jetzt der erste Schritt entfällt, bleibt $\partial \widetilde{G}$ bei den Deformationen von $\tilde{G}$ fest, d.h. aber, daß wir gerade die gewünschte Normierung von $G$ in $M$ erhalten.

(2.9) Satz. Sei $p: M \rightarrow B$ ein $S^{1}$-Bündel. Sei B orientierbar. $G$ sei ein System von Flächen in $M$. Es sei $p \mid G$ lokal homöomorph. $G^{\prime}$ sei eine Komponente von G. $p \mid G^{\prime}$ habe die Blätterzahl $\alpha$.

Dann gibt es eine (semilineare) Abbildung $H: M \times S^{1} \rightarrow M$ mit folgenden Eigenschaften (dabei werde die 1-Sphäre $S^{1}$ identifiziert mit den reellen Zahlen modulo den ganzen Zahlen):

(2.9.1) $H \mid x \times S^{1}$, für $x \in M$, ist ein Homöomorphismus von $S^{1}$ auf die $x$ enthaltende Faser.

(2.9.2) $H(y)=H \mid M \times y$, für $y \in S^{1}$, ist ein Homöomorphismus von $M$ auf $M$. 
(2.9.3) $H$ ist additiv in $y, d . h . H\left(y_{1}+y_{2}\right)=H\left(y_{1}\right) \circ H\left(y_{2}\right)$.

(2.9.4) $H(1 / \alpha) \mid G^{\prime}$ erzeugt die Decktransformationengruppe für $p \mid G^{\prime}$.

(2.9.5) Eine Bündelprojektion von $M$ auf die 1-Sphäre (die diesmal identifiziert wird mit den reellen Zahlen modulo $1 / \alpha$ ) ist dadurch definiert, daß einem Punkt $x$ in $M$ genau dann die Restklasse $y$ mod. 1/ $\alpha$ zugeordnet wird, wenn $x \in H(y)\left(G^{\prime}\right)$.

(2.9.6) Sämtliche Komponenten von $G$ sind Fasern der in (2.9.5) definierten Faserung.

Beweis. Sei $\omega$ die Anzahl der Komponenten von G. Die Anzahl der in einer Faser liegenden Punkte von $G$ hängt nicht von der Faser ab; sie ist $=\omega \alpha$. Wir orientieren $M$ und $B$ : Dann ist eine Orientierung der Fasern eindeutig definiert. Wir schneiden $M$ an $G$ auf; $M$ zerfällt dadurch in Schichten, in denen die Faserung von $M$ eine Faserung als Linienbündel mit orientierten Fasern, also als Produktbündel induziert.

Wir parametrisieren diese Linienbündel als $G^{\prime \prime} \times\left[0,(\omega \alpha)^{-1}\right]$, wobei $G^{\prime \prime} \times 0$ die „untere“ Randfläche sei.

Für $x \in G^{\prime \prime}$ und $0 \leqq y \leqq(\omega \alpha)^{-1}$ definieren wir $H(y)(x \times 0)=x \times y$.

Wegen (2.9.3) ist dann $H$ wohldefiniert. - Die übrigen Eigenschaften folgen unmittelbar aus der Konstruktion.

(2.10) Lemma. Sei $M$ das Produktbündel über einer gelochten 2Sphäre. $M$ habe $m+1$ Randflächen.

$F$ und $G$ seien inkompressible Flächensysteme in $M$, die zum Typ (2.8.2) gehören.

In jeder der ersten $m$ Randflächen von $M$ seien die darin liegenden Randkurven von $F$ und $G$ zueinander homolog.

Dann gibt es eine Deformation von $F$, nach deren Ausfiührung $F \cap G=\emptyset$.

(2.11) Folgerung. Jede Komponente von $F$ ist isotop zu jeder Komponente von $G$. - Denn nach (2.10) dürfen wir annehmen, daB $F$ und $G$ disjunkt sind, daß also $F \cup G$ ein Flächensystem vom Typ (2.8.2) ist: Die Deformation wurde in (2.9) angegeben.

Beweis von (2.10). Da die Komponenten von $F$ bzw. die von $G$ untereinander parallel sind, (2.9), können wir ohne Einschränkung der Allgemeinheit annehmen, da $F$ und $G$ nur je eine Komponente haben.

Ein Argument aus dem Beweis zu (4.2), (,,$\alpha$ ist das kleinste gemeinsame Vielfache von je $n-1$ der $\left.\alpha_{j}{ }^{\prime \prime}\right)$, zeigt, daß die beiden Überlagerungen $p \mid F$ und $p \mid G$ von $B$ gleiche Blätterzahl haben.

Wir gehen zurück in den Beweis von (2.8), nach dem dritten Normierungsschritt. $T_{1}, \ldots, T_{m+1}$ seien die Randflächen von $M$. Wir können uns das System der vertikalen Kreisringe $C=C_{1} \cup \cdots \cup C_{m}$ so gewählt denken, daß je eine Randkurve von $C_{j}$ in $T_{j}$ und $T_{j+1}$ liegt. 
Sei nun $F$ bereits so deformiert, daß

$$
F \cap G \cap\left(T_{1} \cup C_{1} \cup T_{2} \cup \cdots \cup C_{j-2} \cup T_{j-1}\right)=\emptyset
$$

für ein $j \leqq m$, und daß in $T_{j-1}$ je zwei benachbarte Randkurven von $G$ durch eine Randkurve von $F$ getrennt werden. Da $T_{j} z u$ den ersten $m$ Randflächen von $M$ gehört, sind die in $T_{j}$ liegenden Randkurven von $F$ und $G$ zueinander isotop; da $F$ und $G$ gleiche Blätterzahl über $B$ haben, liegen in $T_{j}$ also auch gleich viele Randkurven von $F$ und $G$. Folglich können wir durch eine ,vertikale“" Isotopie (d.h. eine solche, die jeden Punkt nur innerhalb seiner Faser bewegt) von $F$ in der Nähe von $C_{j-1} \cup T_{j}$ erreichen, daß (zusätzlich zur Induktionsvoraussetzung) gilt: Die in $T_{j}$ liegenden Randkurven von $F$ und $G$ sind zueinander punktfremd und trennen sich gegenseitig, und einer der Bögen $F \cap C_{j-1}$ ist punktfremd zu $G$. Mit einem „Kästchenargument" folgt nun, daß die Endpunkte der übrigen Bögen $F \cap C_{j-1}$ so liegen, daß wir eine vertikale Isotopie von $F$ in der Nähe von $C_{j-1}$, aber konstant auf $\partial C_{j-1}$, finden können, so daß schließlich $F \cap G \cap\left(T_{1} \cup C_{1} \cup T_{2} \cup \cdots \cup C_{j-1} \cup T_{j}\right)=\emptyset$.

Mit demselben Argument können wir für $T_{m+1}$ schließen, sobald wir wissen, daß eine in $T_{m+1}$ liegende Randkurve $k$ von $F$ in $T_{m+1}$ homolog ist zu einer in $T_{m+1}$ liegenden Randkurve $l$ von $G$. - Angenommen $k$ und $l$ seien nicht homolog, dann haben sie von null verschiedene Schnittzahl. Wir orientieren $F$ und $G$ und versehen die Randkurven mit der induzierten Orientierung. Da $p \mid F$ und $p \mid G$ Überlagerungen sind, sind die Homologieklassen $\left[F \cap T_{m+1}\right]$ und $\left[G \cap T_{m+1}\right]$ von null verschiedene Vielfache von [k] bzw. [l], also $\left[F \cap T_{m+1}\right] \cdot\left[G \cap T_{m+1}\right] \neq 0$, (Schnittzahl in $\left.T_{m+1}\right)$. Es folgt, daß auch die Schnittzahl in $\partial M,\left[F \cap T_{m+1}\right] \cdot[\partial G]$ nicht null ist; die ist aber bis höchstens aufs Vorzeichen gleich der Schnittzahl von $\left[F \cap T_{m+1}\right] \in H_{1}(M)$ mit $[G] \in H_{2}(M, \partial M)$. Da für $j \leqq m$ der Durchschnitt $F \cap G \cap T_{j}$ leer ist, ist die letzte Schnittzahl gleich der von $[\partial F] \in H_{1}(M)$ mit $[G] \in H_{2}(M, \partial M)$, die ist aber gleich null.

Gehen wir wieder in den Beweis von (2.8) zurück, so sehen wir, daß unser Problem reduziert ist auf folgende Aufgabe: $\widetilde{F}$ und $\widetilde{G}$ seien Systeme von Meridianflächen in einem Vollring $\tilde{M}$; es sei $\partial \tilde{F} \cap \partial \tilde{G}=\emptyset$. Dann sei $\tilde{F}$ bei festgehaltenem Rand so zu deformieren, da $\beta \tilde{G}$ nicht mehr getroffen wird. - Daß das geht, folgt aus (2.2).

\section{Das $\boldsymbol{S}^{\mathbf{1}}$-Bündel über dem Möbiusband}

In diesem Paragraphen ist $p: M \rightarrow B$ das Produktbündel über der 3-fach gelochten 2-Sphäre. Die Randflächen von $M$ bezeichnen wir mit $T_{1}, T_{2}, T_{3}$.

Sei $\tilde{G}$ eine Fläche in $M$ mit den Eigenschaften: $p \mid \tilde{G}$ ist eine zweiblättrige Uberlagerung; in $T_{1}$ und $T_{2}$ liegt je eine Randkurve von $\tilde{G}$; in $T_{3}$ liegen zwei Randkurven von $\tilde{G}$. (Es gibt unendlich viele, paarweise 
nicht isotope Flächen dieser Art in $M$; wir nehmen irgendeine.) $\tilde{G}$ ist eine 4-fach gelochte 2-Sphäre.

Wir konstruieren die Mannigfaltigkeit $Q$ folgendermaßen: Seien $V_{1}$ und $V_{2}$ Vollringe. Wir kleben $V_{1}$ und $V_{2}$ an $M$ an, indem wir $\partial V_{j}$ so mit $T_{j}, j=1,2$, identifizieren, daß die Kurve $\widetilde{G} \cap T_{j}$ zusammenfällt mit der Randkurve einer Meridianfläche von $V_{j}$.

Aus jedem der $V_{j}$ kleben wir eine Meridianfläche an $\tilde{G}$ an, und erhalten so die Fläche $G$ in $Q ; G$ ist ein Kreisring.

(3.1) Lemma. $Q$ ist homöomorph zu dem $S^{1}$-Bündel mit orientierbarem Totalraum über dem Möbiusband.

Beweis. Nach (2.9) ist $M$ ein Bündel über $S^{1}$ mit $\tilde{G}$ als typischer Faser. Diese Faserung induziert eine Faserung der $\partial V_{j}$ durch Meridiankurven, und diese wiederum können wir erweitern zu einer Faserung der $V_{j}$ durch Meridianflächen. Durch Zusammensetzen der Fasern erhalten wir eine Kreisring-Faserung von $Q$ über $S^{1}$ (mit $G$ als typischer Faser). Es gibt nur zwei Kreisring-Bündel über $S^{1}$ mit orientierbarem Totalraum: Das eine läßt sich in natürlicher Weise fasern als $S^{1}$-Bündel über dem Möbiusband, das andere als $S^{1}$-Bündel über dem Kreisring. $Q$ ist nicht das letztere, da $Q$ nur eine Randfläche hat.

In $B$ sei $k$ ein Bogen, dessen Endpunkte in $p\left(T_{3}\right)$ liegen, und der $p\left(T_{1}\right)$ und $p\left(T_{2}\right)$ trennt. Sei $K$ der Kreisring $K=p^{-1}(k)$ in $M$. Bei der Einbettung von $M$ in $Q$ wird $K$ zu einem Kreisring in $Q$.

(3.2) Lemma. Sei $F$ eine inkompressible Fläche in $Q ; F$ sei nicht randparallel. Dann ist $F$ ein Kreisring, und $F$ ist entweder isotop zu dem Kreisring $K$ oder zu dem Kreisring $G$.

Beweis. Nach (3.1) und (2.7) ist $Q$ irreduzibel. Daher können wir (1.9) anwenden, indem wir annehmen, daß $F \cap\left(T_{1} \cup T_{2}\right)$ aus möglichst wenig Kurven besteht.

1. Fall. $F$ liegt in $M$. Da $F$ nicht alle Randflächen von $M$ trifft, ist es vom Typ (2.8.1), also in $M$ isotop zu einem vertikalen Torus oder Kreisring. Ein vertikaler Torus in $M$ ist parallel zu $T_{1}$ oder $T_{2}$ oder $T_{3}$ : Die beiden ersten Typen sind kompressibel in $Q$, der dritte Typ ist rand-parallel in $Q$. Ein vertikaler Kreisring in $M$, dessen Rand in $T_{3}$ liegt, ist entweder isotop zu $K$ oder rand-parallel in $M$ und a fortiori in $Q$.

2. Fall. $F \cap\left(T_{1} \cup T_{2}\right) \neq \emptyset$. Nach (1.9) sind die Flächensysteme $F \cap V_{1}$ in $V_{1}$ und $F \cap V_{2}$ in $V_{2}$ inkompressibel und nicht rand-parallel, sie bestehen nach (2.3) aus Meridianflächen. Es ist also einerseits $\tilde{F}=F \cap M$ zusammenhängend, andererseits jede Kurve $\tilde{F} \cap T_{j}$ in $T_{j}$ homolog zu einer Kurve $\tilde{G} \cap T_{j}$, für $j=1,2$. Nach (2.11) ist also $\tilde{F}$ in $M$ isotop zu $\tilde{G}$ : Wir führen die Deformation aus, und können sie dann wegen (2.2) auf die $F \cap V_{j}$ ausdehnen. 
(3.3) Lemma. Sei $p^{\prime}: M^{\prime} \rightarrow B^{\prime}$ das $S^{1}$-Bündel über dem Möbiusband. Bis auf Isotopie gibt es genau zwei inkompressible und nicht rand-parallele Flächen, $F_{1}$ und $F_{2}$, in $M^{\prime}$.

$F_{1}$ ist ein vertikaler Kreisring; $F_{1}$ zerlegt $M^{\prime}$ nicht.

$p^{\prime} \mid F_{2}$ ist eine zweiblättrige Überlagerung; die Randkurven von $F_{2}$ sind horizontal; $F_{2}$ zerlegt $M^{\prime}$.

Unter dem Homöomorphismus (3.1) entspricht $F_{1}$ dem Kreisring $G$ aus (3.2), und $F_{2}$ dem Kreisring $K$ aus (3.2).

Es gibt eine Einbettung von $B^{\prime}$ als Schnitt in $M^{\prime}$. Dabei ist $\partial B^{\prime}$ bis auf Isotopie eindeutig bestimmt, (unabhängig auch davon, wie $M^{\prime}$ als $S^{1}$ Bündel über dem Möbiusband gefasert ist). $\partial B^{\prime}$ ist isotop zu den Randkurven von $F_{2}$.

Beweis. Wegen (3.2) genügt es, die Existenz von $F_{1}$ und $F_{2}$ nachzuweisen. - Der inkompressible vertikale Kreisring $F_{1}$ wurde im Beweis zu (2.7) angegeben; er ist nicht rand-parallel, da er nicht zerlegt. - Man konstruiert $M^{\prime}$ am bequemsten aus dem cartesischen Produkt eines Streifens mit der 1-Sphäre, indem man den Streifen zum Möbiusband schließt und dabei den $S^{1}$-Faktor spiegelt: Das liefert den Schnitt gleich mit. Sei also $B^{\prime}$ als Schnitt in $M^{\prime}$ eingebettet. Sei $U\left(B^{\prime}\right)$ eine reguläre Umgebung von $B^{\prime}$ in $M^{\prime}$. Es sei dann $F_{2}=\left(\partial U\left(B^{\prime}\right)-\partial M^{\prime}\right) . F_{2}$ ist inkompressibel nach dem Argument aus dem Beweis zu (2.7). Wäre $F_{2}$ parallel zu einem Kreisring in $\partial M^{\prime}$, dann berandete $F_{2}$ zusammen mit diesem Kreisring einen Vollring, und es gäbe in diesem Vollring eine Meridianfläche, die $F_{2}$ in nur einem Bogen träfe: Das ist aber hier nicht möglich, da die Inklusionen $H_{1}\left(F_{2}\right) \rightarrow H_{1}\left(U\left(B^{\prime}\right)\right)$ und $H_{1}\left(F_{2}\right) \rightarrow H_{1}\left(\left(M^{\prime}-U\left(B^{\prime}\right)\right)\right)$ beide den Index 2 haben. - Eine Faser in $\partial M^{\prime}$ trifft $\partial F_{2}$ in zwei Punkten; da sie beide Randkurven trifft, sind die Randkurven horizontal.

Daß $F_{1}$ dem Kreisring $G$ aus (3.2) und $F_{2}$ dem Kreisring $K$ aus (3.2) entspricht, folgt daraus, da $F_{1}$ und $G$ nicht zerlegen, und daß $F_{2}$ und $K$ zerlegen.

Nach Konstruktion von $F_{2}$ ist $\partial B^{\prime}$ in $\partial M^{\prime}$ isotop zu den Randkurven von $F_{2}$. Da jede Einbettung von $B^{\prime}$ als Schnitt in $M^{\prime}$ (auch als Schnitt zu einer andern Faserung) einen Kreisring vom Typ $F_{2}$ liefert, und da $F_{2}$ bis auf Isotopie eindeutig bestimmt ist, folgt die Behauptung über $\partial B^{\prime}$. (Aber es gibt zwei Einbettungen von $B^{\prime}$ als Schnitt in $M^{\prime}$, die nicht einmal homotop sind.)

\section{Planare Flächen in $\boldsymbol{S}^{\mathbf{1}}$-Bündeln}

(4.1) Definition. Eine Fläche heißt ,planar", wenn sie das Geschlecht 0 hat, d.h. wenn sie in die 2-Sphäre eingebettet werden kann.

(4.2) Lemma. $p: M \rightarrow B$ sei ein $S^{1}$-Bündel. Es sei B nicht die 2-Sphäre, die projektive Ebene oder das 2-Element. 
$F$ sei eine inkompressible und nicht rand-parallele Fläche in $M . F$ sei planar. Dann gibt es eine Deformation von $F$, so daß hinterher einer der drei folgenden Fälle vorliegt:

(4.2.1) $F$ ist ein vertikaler Kreisring.

(4.2.2) $B$ ist die (mehrfach) gelochte 2-Sphäre. M hat $n$ Randflächen, und es gibt $n-2$ Randflächen von $M$, so daß die darin liegenden Randkurven von $F$ horizontal sind.

(4.2.3) B ist die (mehrfach) gelochte projektive Ebene, und jede Randkurve von $F$ ist horizontal.

Beweis. Wir dürfen annehmen, daß $F$ von einer der beiden in Satz (2.8) beschriebenen Formen ist. Die erste ist oben aufgeführt, also nehmen wir an, $p \mid F: F \rightarrow B$ ist eine Überlagerung.

$p \mid F$ hat endliche Blätterzahl. $H_{1}(\partial F) \rightarrow H_{1}(F)$ ist surjektiv, da $F$ planar ist. Also hat $H_{1}(\partial B) \rightarrow H_{1}(B)$ endlichen Cokern; mit anderen Worten: Die Fläche, die aus $B$ durch Ausfüllen der Löcher mit 2-Elementen entsteht, hat endliche 1. Homologiegruppe; sie ist daher eine 2-Sphäre oder projektive Ebene.

Wir behandeln zunächst den Fall, daß $B$ eine mindestens dreifach gelochte 2-Sphäre ist.

Die Überlagerung $p \mid F: F \rightarrow B$ ist regulär. Die Decktransformationengruppe ist zyklisch, $\alpha$ sei die Ordnung. Da $Z_{\alpha}$, die zyklische Gruppe der Ordnung $\alpha$, eine abelsche Gruppe ist, ist $p \mid F: F \rightarrow B$ bis auf Äquivalenz gekennzeichnet durch den Homomorphismus $H_{1}(B) \rightarrow Z_{\alpha}$, den wir durch Faktorisieren von $\pi_{1}(B) \rightarrow Z_{\alpha}$ erhalten; dieser Homomorphismus ist surjektiv.

Mit $a_{j}$ bezeichnen wir die $j$-te Randkurve von $B, j=1, \ldots, n$, und mit demselben Symbol das von $a_{j}$ repräsentierte Element von $H_{1}(B)$. Bei geeigneter Orientierung der $a_{j}$ gilt dann:

$$
H_{1}(B) \approx\left\{a_{1}, \ldots, a_{n} ; a_{1}+\cdots+a_{n}=0\right\} .
$$

Mit $\gamma_{j}$ bezeichnen wir die Anzahl der über $a_{j}$ liegenden Randkurven von $F . \gamma_{j}$ teilt $\alpha$, und $\alpha_{j}=\alpha / \gamma_{j}$ ist die Ordnung, mit der $a_{j}$ von einer darüberliegenden Randkurve von $F$ überlagert wird.

Sei $\bar{a}_{j}$ das Bild von $a_{j}$ unter dem Homomorphismus $H_{1}(B) \rightarrow Z_{\alpha}$. Die von $\bar{a}_{j}$ erzeugte Untergruppe hat die Ordnung $\alpha_{j}$ und den Index $\gamma_{j}$.

$H_{1}(B)$ wird von je $n-1$ der $a_{j}$ erzeugt, daher $Z_{\alpha}$ von je $n-1$ der $\vec{a}_{j}$. Daher haben keine $n-1$ der $\gamma_{j}$ einen von 1 verschiedenen gemeinsamen Teiler, oder mit anderen Worten: $\alpha$ ist das kleinste gemeinsame Vielfache von je $n-1 \operatorname{der} \alpha_{j}$.

Unsere Behauptung ist zunächst, daß für mindestens eine Randfläche von $M$ die darin liegenden Randkurven von $F$ horizontal sind; das ist äquivalent zu der Behauptung, daß $\alpha_{j}=1$ für mindestens ein $j$. 
Bezeichne $\chi$ die Eulersche Charakteristik. Es ist $\chi(F)=\alpha \chi(B)=$ $\alpha(2-n)$, andererseits $\chi(F)=2-($ Anzahl der Randkurven von $F)=$ $2-\left(\gamma_{1}+\cdots+\gamma_{n}\right)$, also $\alpha(n-2)+2-\sum \gamma_{j}=0$.

Sei zunächst $n \geqq 4$, und es werde angenommen, da $\beta \quad \alpha_{j}>1$, also $\gamma_{j} \leqq \alpha / 2$ für alle $j$. Es folgte, daß $\alpha(n-2)+2-\sum \gamma_{j} \geqq \alpha(n-2)+2-n \alpha / 2=$ $\alpha(n / 2-2)+2>0$, was der obigen Gleichung widerspricht.

Für $n=3$ lautet die Gleichung: $\gamma_{1}+\gamma_{2}+\gamma_{3}=\alpha+2$. Nach dem oben Gezeigten sind die $\gamma_{j}$ paarweise teilerfremd. Da $\gamma_{j}$ Teiler von $\alpha$ ist, folgt $\gamma_{1} \gamma_{2} \gamma_{3} \leqq \alpha$, und schließlich $\gamma_{1}+\gamma_{2}+\gamma_{3} \geqq \gamma_{1} \gamma_{2} \gamma_{3}+2$. Nachprüfen zeigt, da $\beta$ diese Ungleichung nur erfüllbar ist, wenn mindestens zwei der $\gamma_{j}$, etwa $\gamma_{1}$ und $\gamma_{2}=1$ sind. Es ist also $1+1+\gamma_{3}=\alpha+2$; daher $\alpha_{3}=\alpha / \gamma_{3}=1$.

Die vollständige Behauptung folgt jetzt durch Induktion über die Anzahl der Randflächen: indem wir die Randfläche, deren Existenz wir soeben nachgewiesen haben, in der richtigen Weise mit einem Vollring verschließen, $F$ durch Meridianflächen aus diesem Vollring ergänzen, und nun die Induktionsvoraussetzung anwenden.

Sei nun $B$ die gelochte projektive Ebene. Hat $M$ nur eine Randfläche, so ist unsere Behauptung bereits in (3.3) bewiesen. $M$ habe also die Randflächen $T_{1}, \ldots, T_{n}, n \geqq 2$. Seien $C_{1}, \ldots, C_{n-1}$ disjunkte vertikale Kreisringe in $M$, so daß je eine Randkurve von $C_{j}$ in $T_{j}$ und $T_{j+1}$ liegt. Sei

$$
N=U\left(T_{1} \cup C_{1} \cup T_{2} \cup \cdots \cup C_{n-1} \cup T_{n}\right)
$$

eine vertikale reguläre Umgebung. $N$, mit der von $M$ induzierten Faserung, ist das Produktbündel über der $(n+1)$-fach gelochten 2-Sphäre; $(M-N)$ ist das $S^{1}$-Bündel über dem Möbiusband.

Wir wenden Satz (1.9) auf $F$ an, indem wir annehmen, daß der Durchschnitt mit der Fläche $N \cap \overline{(}(M-N)=\partial \overline{(}(M-N)$ aus möglichst wenig Kurven besteht. Nach (3.3) besteht dann $F \cap \overline{(}(M-N)$ aus Kreisringen, deren Randkurven in $\partial(M-N)$ entweder isotop zu vertikalen oder zu horizontalen Kurven sind. Sind diese Kurven isotop zu vertikalen Kurven, so zeigt eine Anwendung von (2.8) auf $F \cap N$, daß die $\partial F$ in $\partial M$ isotop zu vertikalen Kurven sind, und Nachschauen in (2.8) zeigt, da $F$ in $M$ isotop zu einem vertikalen Kreisring ist.

Also nehmen wir an, die $F \cap \partial \overrightarrow{(M}-N)$ sind horizontal: Ist nun eine Komponente von $F \cap N$ nicht isotop in $N$ zu einer horizontalen Fläche, so trifft das auch für alle andern zu, da $F \cap N$ in $N$ ein Flächensystem vom Typ (2.8.2) ist; also hat in diesem Falle jede Komponente von $F \cap N$ mindestens zwei in $N \cap(M-N)$ liegende Randkurven.

Schauen wir nun nach, wie $F$ aus den Teilen $F \cap N$ und den Kreisringen $F \cap(M-N)$ zusammengesetzt ist, so finden wir zwei Möglichkeiten: Entweder wir beginnen mit einer Komponente aus $F \cap N$, hängen an sie einen Kreisring aus $F \cap(M-N)$ an, an diesen wieder eine andere Komponente 
aus $F \cap N$, daran einen andern Kreisring aus $F \cap(M-N)$, usw. ohne Ende: Oder aber, irgendwann sind alle in $N \cap \overline{(}(M-N)$ liegenden Randkurven der benutzten Teile wirklich aufgebraucht, dann hat aber $F$ sicher nicht das Geschlecht 0 .

\section{Homöomorphismen von $\boldsymbol{S}^{\mathbf{1} \text {-Bündeln }}$}

(5.1) Lemma. Sei $C$ ein Kreisring. $C$ sei auf zwei Arten als $S^{1}$-Bündel gefasert, d.h. es seien Bündelprojektionen $p_{j}: C \rightarrow I$ gegeben, $j=1$, 2. Dann gibt es eine isotope Deformation $\psi_{\tau}, 0 \leqq \tau \leqq 1$, von $C$, die die eine Faserung in die andere überführt; die Deformation kann so gewählt werden, daß sie auf $\partial C$ konstant ist.

Beweis. Da es nur ein $S^{1}$-Bündel über $I$ gibt, gibt es einen Homöomorphismus $\varphi: C \rightarrow C$, der die eine Faserung in die andere überführt; $p_{1} \circ \varphi$ $=p_{2}$. Wir können $\varphi$ so wählen, $\mathrm{da} \beta|\partial C=\mathrm{id}| \partial C$. Unter der Bedingung $\varphi|\partial C=\mathrm{id}| \partial C$ ist für einen Bogen $l$, der die beiden Randkurven von $C$ verbindet, eine „Schnittzahl“ von $l$ mit $\varphi(l)$ definiert. Wir können $\varphi$ so wählen, daß diese Schnittzahl null ist. $\varphi$ ist dann in die Identität deformierbar mit einer Deformation, die auf $\partial C$ konstant ist. Wir setzen $\psi_{1}=\varphi$, und nehmen als $\psi_{t}$ die inverse der soeben erwähnten Deformation.

(5.2) Lemma. Ist ein Torus auf zwei Arten gefasert, und ist eine Faser $k$ der einen Faserung homolog zu einer Faser $k^{\prime}$ der andern Faserung, so gibt es eine Deformation des Torus, die die eine Faserung in die andere überführt.

Beweis. Wir deformieren die zweite Faserung so, daß $k=k^{\prime}$, schneiden dann an $k$ auf, und wenden (5.1) an.

(5.3) Lemma. Sei der Vollring $V$ auf zwei Arten als $S^{1}$-Bündel gefasert, aber so, daß die induzierten Faserungen auf $\partial V$ übereinstimmen. Dann gibt es eine auf $\partial V$ konstante isotope Deformation von $V$, die die eine Faserung in die andere überführt.

Beweis. Es gibt einen Homöomorphismus $\varphi: V \rightarrow V$, der die eine Faserung in die andere überführt. $\varphi$ kann so gewählt werden, daß $\varphi|\partial V=\mathrm{id}| \partial V .(*)$ Es gibt dann eine auf $\partial V$ konstante isotope Deformation von $\varphi$ in die Identität. Mit dieser Deformation führen wir die zweite Faserung in die erste über.

Beweis von (*). Sei $F$ eine Meridianfläche in $V$. Nach (2.2) gibt es eine auf $\partial V$ konstante Deformation von $\varphi$, so $\mathrm{da} B \varphi(F)=F$. Es gibt dann weiter eine auf $\partial V$ konstante Deformation von $\varphi$, die auf $F$ eine Deformation von $\varphi \mid F$ nach id $\mid F$ induziert. - Schneiden wir nun $V$ an $F$ auf, so ist unser Problem darauf zurückgeführt, ob ein Homöomorphismus eines 3-Elements auf sich, der auf dem Rande die Identität ist, in die 
Identität deformierbar ist mit einer auf dem Rande konstanten Deformation.

(5.4) Bemerkung. Lemma (5.3) bleibt richtig, wenn man die Worte ,als $S^{1}$-Bündel" ersetzt durch, im Seifertschen Sinne". - Im Beweis hat man nur die Klassifikation von $S^{1}$-Bündeln über dem 2-Element zu ersetzen durch die Tatsache, daß im Seifertschen Sinne gefaserte Vollringe dann fasertreu homöomorph sind, wenn es einen Homöomorphismus der Randflächen aufeinander gibt, der Fasern in Fasern und Meridiankurven in Meridiankurven überführt, [12], S. 153-154.

(5.5) Satz. $p: M \rightarrow B$ und $p^{\prime}: M^{\prime} \rightarrow B^{\prime}$ seien $S^{1}$-Bündel. $B$ oder $B^{\prime}$ sei nicht: 2-Sphäre, projektive Ebene, 2-Element oder Kreisring. Von den Bündeln über dem Torus sei $S^{1} \times S^{1} \times S^{1}$ ausgeschlossen, und von den Bündeln über dem Kleinschen Schlauch dasjenige, das einen Schnitt besitzt.

$\varphi: M \rightarrow M^{\prime}$ sei ein Homöomorphismus.

Dann gibt es einen Homöomorphismus $\psi: M \rightarrow M^{\prime}$ mit den Eigenschaften

a) $\psi$ ist isotop zu $\varphi$.

b) Es gibt einen Homöomorphismus $p(\psi): B \rightarrow B^{\prime}$, so daß $p^{\prime} \circ \psi=$ $p(\psi) \circ p ;$ mit anderen Worten: $\psi$ bildet jede Faser auf eine Faser ab.

Beweis. Sei zunächst $\partial M \neq \emptyset$.

Sei $C$ ein System vertikaler Kreisringe, so da $B$ von $C$ in einen Vollring aufgeschnitten wird. Keine Komponente von $C$ ist kompressibel oder rand-parallel (das erste im Beweis zu (2.7) gezeigt, das zweite ist klar, da $C$ nicht zerlegt). Also ist auch keine Komponente von $\varphi(C)$ kompressibel oder rand-parallel in $M^{\prime}$. Daher können wir $\varphi$ so deformieren, daß $\varphi(C)$ vertikal wird: Ist $M^{\prime}$ nicht das $S^{1}$-Bündel über dem Möbiusband, so folgt das unmittelbar aus (4.2); ist $M^{\prime}$ dieses Bündel (und dann aus Homologiegründen auch $M$ ), so zeigt (3.3), daß ein vertikaler Kreisring nur auf einen zu einem vertikalen isotopen abgebildet werden kann.

Wir wenden nun (5.1) an auf die Kreisringe $\varphi(C)$ und auf die Kreisringe, in die $\partial M^{\prime}$ durch $\varphi(\partial C)$ zerschnitten wird, und finden, daß die durch $\varphi$ übertragene Faserung deformiert werden kann in die durch die Einbettung induzierte Faserung, durch eine Deformation dieser Kreisringe, die auf $\varphi(\partial C)$ konstant ist.

$\mathrm{Da}$ es eine Deformation von $\varphi$ gibt, die die gerade beschriebene Deformation von $\varphi(C \cup \partial M)$ induziert, und da wir die Normierung auf eine Umgebung erweitern können, haben wir: Es gibt eine Deformation von $\varphi$, nach deren Ausführung $\varphi$ jede Faser aus einer vertikalen Umgebung $U(C \cup \partial M)$ auf eine Faser abbildet (d.h. auf eine Faser der durch die Einbettung von $\varphi(U(C \cup \partial M))$ in $M^{\prime}$ induzierten Faserung).

Zum Schluß wenden wir (5.3) an und absorbieren die Isotopie in eine Deformation von $\varphi$. 
Sei $M$ geschlossen. Da $B$ nicht die 2-Sphäre oder projektive Ebene ist, gibt es in $M$ einen nicht-zerlegenden vertikalen Torus $T$; nach (2.6) ist $T$ inkompressibel.

1. Fall. $\varphi(T)$ ist in $M^{\prime}$ nicht isotop zu einem vertikalen Torus. Nach (2.8) dürfen wir annehmen, daß $p^{\prime} \mid \varphi(T)$ eine Überlagerung ist. Folglich ist $B^{\prime}$ ein Torus oder Kleinscher Schlauch.

Sei $T^{\prime}$ ein nicht-zerlegender vertikaler Torus in $M^{\prime}$. Von den Kreisringen, in die $\varphi(T)$ durch $T^{\prime}$ zerschnitten wird, sei $F$ einer. Die Kurven $\partial F$ sind parallel in $T^{\prime}$; sie sind nicht vertikal.

Das Bündel $M^{\prime}$ können wir beschreiben durch eine Verklebung der Randflächen von $T^{\prime} \times I$, also durch einen Isomorphismus $H_{1}\left(T^{\prime}\right) \rightarrow$ $H_{1}\left(T^{\prime}\right)$. Sei $z_{1}, z_{2}$ ein Erzeugendensystem von $H_{1}\left(T^{\prime}\right)$, dabei sei $z_{1}$ die Homologieklasse einer orientierten Faser. Dann hat der Isomorphismus die Form $\left(z_{1}, z_{2}\right) \rightarrow\left(\varepsilon z_{1}, \varepsilon z_{2}+b z_{1}\right)$, mit $\varepsilon=+1$, bzw. -1 , wenn $B^{\prime}$ der Torus bzw. der Kleinsche Schlauch ist; $b$ ist eine ganze Zahl. Die Existenz des Kreisrings $F$ zeigt, daß $b=0$, daß also $M^{\prime}$ eines der ausgeschlossenen Bündel ist. - Um $M$ zu bestimmen, betrachten wir $\varphi^{-1}\left(T^{\prime}\right)$, und schlieBen (möglicherweise erst im 2. Fall), daß auch $M$ eines der ausgeschlossenen Bündel ist.

2. Fall. $\varphi(T)$ ist in $M^{\prime}$ isotop zu einem vertikalen Torus. Wir führen die Deformation aus. Ist die durch $\varphi$ nach $\varphi(T)$ transportierte Faserung nicht isotop zu der von $M^{\prime}$ induzierten, so folgt aus der für berandete Bündel bereits bewiesenen Behauptung, da $\beta$ die aus $M^{\prime}$ durch Aufschneiden an $\varphi(T)$ erhaltene Mannigfaltigkeit nur Torus $\times$ Intervall sein kann. Wie im ersten Fall folgt, daß $M^{\prime}$ eines der verbotenen Bündel ist.

Also nehmen wir an, daß die beiden Faserungen auf $\varphi(T)$ übereinstimmen. Wir schneiden $M$ und $M^{\prime}$ an $T$ und $\varphi(T)$ auf $\mathrm{zu} M_{1}$ und $M_{1}^{\prime}$. Nach dem ersten Teil können wir den induzierten Homöomorphismus $\varphi_{1}: M_{1} \rightarrow M_{1}^{\prime}$ in einen fasertreuen Homöomorphismus deformieren, und zwar geht das auch noch für den oben nicht erfaßten Fall, daß $M_{1}$ homöomorph ist $\mathrm{zu}$ Torus $\times$ Intervall, da wir ja hier in die Voraussetzungen mit aufnehmen können, daß $\varphi_{1} \mid \partial M_{1}$ fasertreu ist. - Nachprüfen der Argumente aus dem ersten Teil zeigt, daß wir die Deformation von $\varphi_{1}$ so wählen können, daß sie auf $\partial M_{1}$ konstant ist: Also existiert die gesuchte Deformation von $\varphi$.

(5.6) Definition. Sei $p: M \rightarrow B$ ein $S^{1}$-Bündel. $M$ sei orientiert. $T_{1}, \ldots, T_{n}$ seien die Randflächen von $M$; wir versehen die $T_{j}$ mit der von $M$ induzierten Orientierung.

(5.6.1) Ein Koordinatensystem in $T_{j}$ ist ein Paar von Homologieklassen $\left[a_{j}\right]$ und $\left[b_{j}\right]$ in $H_{1}\left(T_{j}\right)$, so daß $\left[a_{j}\right]$ und $\left[b_{j}\right]$ von orientierten Kurven $a_{j}$ und $b_{j}$ in $T_{j}$ repräsentiert werden, und so daß gilt: 
$a_{j}$ ist horizontal; $b_{j}$ ist vertikal; $b_{j}$ und $a_{j}$ haben - in dieser Reihenfolge - die Schnittzahl +1 in $T_{j}$ (oder, was dasselbe ist: Schneiden wir $T_{j}$ an $b_{j} \cup a_{j}$ auf, so erhalten wir ein 2-Element, dessen von $T_{j}$ induzierte Orientierung auf seinem Rand die Orientierung $b_{j} a_{j} b_{j}^{-1} a_{j}^{-1}$ induziert.)

(5.6.2) Sind Koordinatensysteme in allen Randflächen von $M$ gegeben, so heißen sie ein Koordinatensystem für $\partial M$, wenn folgendes gilt:

a) Es gibt eine Einbettung der Basis als Schnitt in $M$, so daß (als Punktmenge) $\partial B=\bigcup a_{j}$.

b) Ist $B$ orientierbar, dann sind die $b_{j}$, gleich orientiert", d.h. in $M$ homolog.

(5.7) Lemma. $Z u$ den unten angegebenen Änderungen eines Koordinatensystems gibt es Homöomorphismen von $M$ auf sich (orientierungsumkehrend in den beiden ersten Fällen, orientierungserhaltend in den übrigen), die diese Änderungen induzieren.

(5.7.1) $b_{j} \rightarrow-b_{j} ; a_{j} \rightarrow+a_{j}$; für alle $j$.

(5.7.2) $b_{j} \rightarrow+b_{j} ; a_{j} \rightarrow-a_{j}$; für alle $j$.

(5.7.3) $b_{j} \rightarrow-b_{j} ; a_{j} \rightarrow-a_{j}$; für alle $j$.

(5.7.4) Wenn $B$ nicht-orientierbar ist, $b_{1} \rightarrow-b_{1} ; a_{1} \rightarrow-a_{1} ; b_{j} \rightarrow+b_{j}$; $a_{j} \rightarrow+a_{j}$; für $j \geqq 2$.

(5.7.5) $b_{1} \rightarrow b_{2} ; a_{1} \rightarrow a_{2} ; b_{2} \rightarrow b_{1} ; a_{2} \rightarrow a_{1} ; b_{j} \rightarrow b_{j} ; a_{j} \rightarrow a_{j}$; für $j \geqq 3$. für $j \geqq 3$.

(5.7.6) $b_{j} \rightarrow b_{j}$; für alle $j ;\left[a_{1}\right] \rightarrow\left[a_{1}\right]+\left[b_{1}\right] ;\left[a_{2}\right] \rightarrow\left[a_{2}\right]-\left[b_{2}\right] ; a_{j} \rightarrow a_{j}$;

Beweis. $z u$ 1) Wir betten die Basis als Schnitt in $M$ ein. Sei $U(B)$ eine reguläre Umgebung von $B$ in $M$, deren Durchschnitt mit jeder Faser aus einem Bogen besteht. Sei $F=(\partial U(B)-\partial M)$. Wir können $B$ und $U(B)$ so wählen, daß jede der Kurven $a_{j}$ eine Randkurve von $F$ ist: Der gesuchte Homöomorphismus spiegelt jede Faser so, daß ihr Durchschnitt mit $F$ fest bleibt.

$z u 2,3,4,5) \mathrm{Zu}$ einem gegebenen Homöomorphismus von $B$ auf sich gibt es einen Homöomorphismus von $M$ auf sich, der diesen induziert: dazu genügt es zu bemerken, daß sich über einem Weg von $B$ die Faserorientierung genau dann umkehrt, wenn sich längs dieses Weges die lokale Orientierung von $B$ umkehrt.

Sei $B^{\prime}$ eine geschlossene Fläche, und seien $D_{1}, \ldots, D_{n}$ 2-Elemente in $B^{\prime}$, so da $B B^{\prime}-\bigcup \stackrel{\circ}{D}_{j}$ homöomorph ist zu $B$. Ist $B$ orientierbar, dann sei $B$ so orientiert, da $B$ in jedem der $a_{j}$ die richtige Orientierung induziert wird; das ist möglich nach den Bedingungen aus (5.6). Ist $B$ nichtorientierbar, so sei jedes der $D_{j}$ so orientiert, daß es in $a_{j}$ die falsche Orientierung induziert. 
Sei $M^{\prime}$ ein $S^{1}$-Bündel über $B^{\prime}$ (mit orientierbarem Totalraum), in das $B^{\prime}$ als Schnitt eingebettet ist; ein solches existiert. Es ist $M^{\prime}-p^{\prime-1}\left(\bigcup \stackrel{\circ}{D}_{j}\right)$ $=M$.

$z u$ 5) Wir wählen eine fasertreue Isotopie von $M^{\prime}$, die $B^{\prime}$ in sich bewegt, und deren Beschränkung auf $B^{\prime}, D_{1}$ und $D_{2}$ vertauscht. Wenn $B^{\prime}$ nicht-orientierbar ist, können wir das so einrichten, daB der Endzustand auf $D_{1} \cup D_{2}$ einen orientierungserhaltenden Homöomorphismus induziert. - Die Beschränkung des Endzustandes der Isotopie von $M^{\prime}$ auf $M$ ist der gesuchte Homöomorphismus.

$z u$ 4) Sei $k$ eine Kurve in $B^{\prime}$, die $D_{1}$ in einem Bogen und kein anderes der $D_{j}$ trifft, so da $B$ sich längs $k$ die lokale Orientierung von $B^{\prime}$ umkehrt. Wir wählen eine Isotopie von $B^{\prime}$, die $D_{1}$ einmal längs $k$ herumführt und die übrigen $D_{j}$ festläßt, und eine fasertreue Isotopie von $M^{\prime}$, die die angegebene Isotopie von $B^{\prime}$ induziert: Deren Endzustand induziert auf $M$ den gesuchten Homöomorphismus.

$z u 2,3)$ Ist $B$ nicht-orientierbar, dann ergibt Anwenden von (4) auf alle Randflächen (3); (3) und (1) ergibt (2).

Ist $B$ orientierbar, dann sei $\varphi$ ein orientierungsumkehrender Homöomorphismus von $B^{\prime}$. Wir dürfen annehmen, daß $\varphi\left(D_{j}\right)=D_{j}$, da wir das durch eine Deformation erreichen können. Sei $\psi: M \rightarrow M$ ein fasertreuer Homöomorphismus, der $B$ in sich überführt, so daß $\psi|B=\varphi| B$. Je nachdem $\psi$ die Orientierung erhält oder nicht, haben wir (3) oder (2),

$z u$ 6) Sei $l$ ein Bogen in $B$, der $p\left(T_{1}\right)$ und $p\left(T_{2}\right)$ verbindet. Sei $l \times I$ eine reguläre Umgebung von $l$ in $B ; l \times I \cap \partial B=\partial l \times I ; l=l \times 1 / 2$; für $x=\ln p\left(T_{1}\right)$ sei $x \times 0 \rightarrow x \times 1$ die Richtung von $a_{1}$. Wir parametrisieren $p^{-1}(l \times I)=l \times I \times S^{1}$ durch $(x, y, z), x \in l, y \in I, z \in 2 \pi I$, so daß wachsendes $z$ die Orientierung von $b_{1}$ liefert.

Der Homöomorphismus ist $(x, y, z) \rightarrow(x, y, z+2 \pi y)$ auf $p^{-1}(l \times I)$, und die Identität außerhalb. Nach Konstruktion ist $\left[a_{1}\right] \rightarrow\left[a_{1}\right]+\left[b_{1}\right]$; wegen der Orientierungsbedingung aus (5.6.1) ist $\varepsilon\left[a_{2}\right] \rightarrow \varepsilon\left[a_{2}\right]-\varepsilon\left[b_{2}\right]$, mit $\varepsilon=+1$ oder -1 .

(5.8) Lemma. Sei $p: M \rightarrow B$ ein $S^{1}$-Bündel. B sei nicht das 2-Element oder der Kreisring.

(5.8.1) Ein Homöomorphismus von $M$ auf sich führt ein Koordinatensystem für $\partial M$ wieder in ein solches über.

(5.8.2) Ein beliebiges Koordinatensystem für $\partial M$ kann aus einem vorgegeben durch Anwendung der in (5.7) angegebenen Homöomorphismen erhalten werden. Gehören die beiden Koordinatensysteme zur gleichen Orientierung von $M$, so braucht man nur orientierungserhaltende Homöomorphismen anzuwenden. 
Beweis. $z u$ (5.8.1) Wegen (5.5) genügt es, die Behauptung für fasertreue Homöomorphismen nachzuweisen: dafür ist sie aber nahezu trivial. (Ist der Homöomorphismus orientierungsumkehrend, dann gehört natürlich das zweite Koordinatensystem zur umgekehrt orientierten Mannigfaltigkeit.)

$z u$ (5.8.2) Sei $\left[a_{j}\right],\left[b_{j}\right]$ das vorgegebene Koordinatensystem und $\left[a_{j}^{\prime}\right],\left[b_{j}^{\prime}\right]$ das andere. - Da der Homöomorphismus (5.7.5) Randflächen vertauscht, dürfen wir annehmen, daß gleich numerierte Paare in derselben Randfläche liegen.

Es ist $\left[b_{j}^{\prime}\right]=\varepsilon_{j}\left[b_{j}\right]$ (diese und die folgenden Homologien sind Homologien in $T_{j}$ ). Ist $B$ orientierbar, dann ist $\varepsilon_{1}=\cdots=\varepsilon_{n}$; also dürfen wir nach (5.7.3) annehmen, daß $\varepsilon_{j}=1$ für alle $j$. Ist $B$ nicht-orientierbar, dann dürfen wir nach (5.7.4) dasselbe annehmen.

Es ist $\left[a_{j}^{\prime}\right]=\alpha_{j}\left[a_{j}\right]+\beta_{j}\left[b_{j}\right]$; da sowohl $\left[a_{j}^{\prime}\right]$ als auch $\left[a_{j}\right]$ mit $\left[b_{j}^{\prime}\right]=\left[b_{j}\right]$ die Schnittzahl \pm 1 haben, ist $\alpha_{j}= \pm 1$. Ist eines der $\alpha_{j}=-1$, dann auch alle andern, und die beiden Koordinatensysteme gehören zu verschiedenen Orientierungen von $M$; wir wenden dann (5.7.2) an. Also nehmen wir an, $\left[a_{j}^{\prime}\right]=\left[a_{j}\right]+\beta_{j}\left[b_{j}\right]$.

Mit Homöomorphismen (5.7.6), die wir jeweils an der $j$-ten, $j \neq 1$, und 1-ten Randfläche ausführen, erreichen wir, daß $\beta_{j}=0$ für $j \geqq 2$. Wir behaupten, da $B$ dann automatisch auch $\beta_{1}=0$ ist: Wir konstruieren $M^{\prime}$ aus $M$ dadurch, daß wir an $T_{2}, \ldots, T_{n}$ Vollinge $V_{2}, \ldots, V_{n}$ so ankleben, daß $a_{j}$ Meridiankurve auf $\partial V_{j}$ wird, $j=2, \ldots, n$; dann ist auch $a_{j}^{\prime}$ Meridiankurve auf $\partial V_{j}$. Also gibt es eine solche Einbettung der Basis als Schnitt in $M^{\prime}$, daß $a_{1}$, und eine solche, daß $a_{1}^{\prime}$ ihre Randkurve ist. - Ist die Basis von $M^{\prime}$ orientierbar, dann ist $\operatorname{ker}\left(H_{1}\left(\partial M^{\prime}\right) \rightarrow H_{1}\left(M^{\prime}\right)\right)$ frei zyklisch und wird erzeugt von $a_{1}$ bzw. $a_{1}^{\prime}$.

Ist die Basis von $M^{\prime}$ nicht-orientierbar, dann sei $M^{\prime \prime}$ die zweiblättrige Überlagerung von $M^{\prime}$, mit orientierbarer Basis. Über $\left(a_{1}, b_{1}\right)$ liegt ein Koordinatensystem für $\partial M^{\prime \prime}:\left(c_{1}, d_{1}\right),\left(c_{2}, d_{2}\right)$; von der Decktransformation wird $\left(c_{1}, d_{1}\right)$ auf $\left(-c_{2},-d_{2}\right)$ abgebildet. Entsprechend liegt über $\left(a_{1}^{\prime}, b_{1}\right)$ ein Koordinatensystem für $\partial M^{\prime \prime}:\left(c_{1}^{\prime}, d_{1}\right),\left(c_{2}^{\prime}, d_{2}\right)$. Es ist $\left[c_{1}^{\prime}\right]=$ $\left[c_{1}\right]+\beta_{1}\left[d_{1}\right]$ und $\left[c_{2}^{\prime}\right]=\left[c_{2}\right]+\beta_{1}\left[d_{2}\right]$. Mit Homöomorphismen (5.7.6) konstruieren wir ein Koordinatensystem $\left(c_{1}^{\prime \prime}, d_{1}\right),\left(c_{2}^{\prime \prime}, d_{2}\right)$, so daß

$$
\begin{aligned}
& {\left[c_{1}^{\prime \prime}\right]=\left[c_{1}^{\prime}\right]+\beta_{1}\left[d_{1}\right]=\left[c_{1}\right]+2 \beta_{1}\left[d_{1}\right]} \\
& {\left[c_{2}^{\prime \prime}\right]=\left[c_{2}^{\prime}\right]-\beta_{1}\left[d_{2}\right]=\left[c_{2}\right] .}
\end{aligned}
$$

Es folgt, daß $\beta_{1}=0$.

Das Literaturverzeichnis folgt in Teil II dieser Arbeit, der im Band 4, Heft 2 erscheinen wird. 